\title{
A statistical study of the force balance and structure in the flux ropes in Mercury's magnetotail
}

J. T. Zhao, ${ }^{1}$ W. J. Sun, ${ }^{2}$ Q. G. Zong, ${ }^{1}$ J. A. Slavin, ${ }^{2}$ X. Z. Zhou, ${ }^{1}$ R. M.

Dewey, $^{2}$ G. K. Poh, ${ }^{3,4}$, and J. M. Raines ${ }^{2}$

Corresponding author: Q. G. Zong, (qgzong@pku.edu.cn) and W. J. Sun, (wjsun@umich.edu).

${ }^{1}$ School of Earth and Space Sciences,

Peking University, Beijing 100871, China.

${ }^{2}$ Department of Climate and Space

Sciences and Engineering, University of

Michigan, Ann Arbor, Michigan 48109,

USA.

${ }^{3}$ Solar System Exploration Division,

NASA Goddard Space Flight Center,

Greenbelt, Maryland 20771, USA

${ }^{4}$ Center for Research and Exploration in

Space Sciences \& Technology II, University

of Maryland Baltimore County, Baltimore,

Maryland 21250, USA

This is the author manuscript accepted for publication and has undergone full peer review but has not been through the copyediting, typesetting, pagination and proofreading process, which may lead to differences between this version and the Version of Record. Please cite this article

D RaAd Ei: Ti 0.1029/2018JA02632May 23, 2019, 11:44pm

D R A F T

This article is protected by copyright. All rights reserved. 
Abstract. This study presents a statistical investigation of the force balance and structures in the flux ropes in Mercury's magnetotail plasma sheet by using the measurements of MErcury Surface,Space ENviroment,GEochemistry,and Ranging (MESSENGER). 168 flux ropes was identified from the 14 hot seasons of MESSENGER from 11 March 2011 to 30 April 2015, and 143 of them show clear magnetic field enhancements with the core field being $\geq 20 \%$ higher than the background magnetic field. The investigation on the force balance of these 143 flux ropes shows that magnetic pressure gradient force cannot be solely balanced by magnetic tension force, implying that thermal plasma pressure gradient force cannot be neglected in the flux ropes. We employ a non-force-free model considering the contribution of thermal pressure to resolve the physical properties of flux ropes in Mercury's magnetotail. 28 flux ropes are obtained through the fitting to the non-force-free model. The flux ropes are found to be consistent with the flattened structures, in which the mean semi-major is $\sim 851 \mathrm{~km}$ and semi-minor is $\sim 333 \mathrm{~km}$, both are several times the local proton inertial length. The average core field is estimated to be $\sim 57.5 \mathrm{nT}$ and flux content is $\sim 0.019 \mathrm{MWb}$, much larger than the previous results obtained from force-free flux rope model. The importance of thermal pressure gradient in the force-balance of the flux ropes and the flattened structure indicate the flux ropes in Mercury's magnetotail plasma sheet are mostly in early stage of the evolution, and still contain enough plasma to affect their magnetic structures. 


\section{Introduction}

Mercury is the innermost planet in the Solar System with an orbital period of only $\sim 88$ Earth solar days. The Mercury's elliptical orbit about the sun has an aphelion of $\sim 0.47 \mathrm{AU}$ (Astronomical Unit, $1 \mathrm{AU}=1.496 \times 10^{8} \mathrm{~km}$ ) and a perihelion of $\sim 0.31$ AU. The proximity of Mercury's orbit to the sun result in it experiencing interplanetary conditions much different from the other planets in the Solar System. For example, the solar wind is hotter, solar wind density is higher, and the interplanetary magnetic field (IMF) is much stronger at Mercury than those at Earth ( 1 AU) [e.g., Russell et al., 1988; Glassmeier, 1997; Slavin et al., 2007]. Observations from Mariner 10 and MErcury Surface, Space ENviroment, GEochemistry, and Ranging (MESSENGER) [Solomon et al., 2001] have revealed that Mercury's internal magnetic field is closely aligned $\left(<5^{\circ}\right)$ with the planet's rotation axis, and has the same polarity as the Earth. However, the magnetic field near Mercury's surface is only $\sim 1 \%$ of Earth's surface field [e.g., Ness et al., 1976; Alexeev et al., 2010; Anderson et al., 2010, 2011]. Due to the higher solar wind pressure, ${ }_{14}$ weaker internal magnetic field, and stronger dayside magnetopause erosion [e.g., Slavin and Holzer, 1979], the subsolar standoff distance for Mercury's magnetopause is only $\sim 0.45 \mathrm{R}_{\mathrm{M}}$, where $\mathrm{R}_{\mathrm{M}} \sim 2440 \mathrm{~km}$ is Mercury's radius, above the surface of the planet [e.g., Winslow et al., 2013; Zhong et al., 2015]. As a result, Mercury itself occupies a much larger fraction of the magnetosphere than Earth, Saturn, and Jupiter [e.g., Jackman et al., 2014].

Mercury's magnetosphere experiences many processes and structures closely related with magnetic reconnection similar to the Earth's magnetosphere, such as the flux transfer 
22 Flux ropes were proposed to be formed between the near and distant neutral lines during Earth's magnetospheric substorm with magnetic loop profiles (or "O-lines") in 1970s ${ }_{28}$ [Schindler, 1974; Hones, 1977]. The formation of magnetic loop topology inside flux ropes would require perfect anti-parallel magnetic field lines (180 separation angle)[Hughes and Sibeck, 1987; Zong et al., 1997, 2004]. However, because a dawn-dusk component in the magnetotail magnetic field is common, magnetic reconnection would generate the flux ropes with helical field line topology [e.g., Hughes and Sibeck, 1987; Slavin et al., 1989; Hesse and Birn, 1991; Moldwin and Hughes, 1991; Zong et al., 1997, 2004]. A statistical survey on the spatial distribution of flux ropes in Mercury's magnetotail showed that flux ropes were more frequently observed on the dawnside plasma sheet than on the duskside [Sun et al., 2016], indicating that the dawnside plasma sheet is more dynamic 37 on dipolarizations and particle energization, including proton and electron, in the near planet region of Mercury [Sun et al., 2017; Dewey et al., 2017; Smith et al., 2017; Poh et al., 2017a].

The flux ropes could be fitted to a force-free flux rope model whose solution is Bessel functions, which give the diameter, core field intensity, and magnetic flux content for the structures [e.g., Lundquist, 1950; Burlaga, 1988; Lepping et al., 1996; Slavin et al., 44 2003]. The underlying assumptions of this force-free model include $\mathrm{J}$ being parallel to 
${ }_{45}$ B everywhere $(\vec{J} \times \vec{B}=0)$ and the flux rope being cylindrical in shape. There are also

46 several flux rope models that consider the nature of non-force-free of flux ropes and the

${ }_{47}$ influence of gradients in plasma pressure. [e.g., Moldwin and Hughes, 1991; Kivelson

${ }_{48}$ and Khurana, 1995; Hidalgo et al., 2002]. In particular, Kivelson and Khurana [1995]

developed models for flux ropes embedded in Harris current sheet, which contain solutions

for both force-free and non-force-free flux ropes. Their models have been successfully

${ }^{51}$ applied in the flux ropes in the Earth's plasma sheet observed during Galileo's Earth

flyby [Kivelson and Khurana, 1995]. In addition, Slavin et al. [2009] and Slavin et al. [2012a] analyzed FTE-type flux ropes at the Mercury's magnetopause using force-free [Lundquist, 1950] and non-force-free [Hidalgo et al., 2002] models.

By employing the force-free flux rope model first developed by Lundquist [1950], DiBraccio et al. [2015] and Smith et al. [2017] conducted statistical studies on the flux ropes in Mercury's magnetotail. Because MESSENGER could not directly resolve the proton bulk flow velocity, both of them assumed a velocity of $\sim 465 \mathrm{~km} / \mathrm{s}$ for the flux ropes, which was an average value of background Alfvén speed. The radius of flux rope was so found to be $\sim 200 \mathrm{~km}$ comparable to the background ion inertial length. The flux content of flux rope was only $\sim 0.002 \mathrm{MWb}$ on average, which was much smaller (by an order of magnitude) than the latterly reported average magnetic flux of dipolaring flux bundles (DFBs) following dipolarization fronts $(\sim 0.06 \mathrm{MWb})$ [Dewey et al., 2018] and two orders of magnitude smaller than the magnetic flux loaded into Mercury's magnetotail during the substorm growth phase ( 0.69 MWb) [Slavin et al., 2010; Imber and Slavin, 2017]. However, the new MMS observations have shown that thermal pressure gradients are ${ }_{67}$ important in newly formed ion-scale flux ropes [Farrugia et al., 2016; Zhao et al., 2016]. 
${ }_{68}$ Because the flux ropes at Mercury are ion-scale, and may have formed only recently, its

${ }_{69}$ force balance within the flux ropes in Mercury's tail may also involve significant plasma

70 pressure gradients. Since force-free model does not consider the contribution of thermal

${ }_{71}$ pressure, if the thermal pressure is significant, thus it may be important to apply a non-

force-free model to the flux ropes in Mercury's tail.

Here, we investigate the force balance within these flux ropes at Mercury, Our results ${ }_{74}$ show that thermal plasma pressure gradients cannot be ignored inside most of the flux ropes. The physical properties of the flux ropes are determined by comparing the results of non-force-free and force-free modeling. This study finds that most of the ion-scale flux ropes observed in Mercury's magnetotail by MESSENGER appear to have formed recently and still contain significant amounts of plasma, which might still be able to affect their magnetic structures.

This paper arranges as follows. In Section 2, the instrumentation and data will be described. In Section 3, at first, we will show a flux rope case study. Secondly, we will statistically investigate the force balance of flux ropes, and then we will describe the s3 non-force-free flux rope model employed in this research. Section 4 will provide detail statistical results for the structure of flux ropes in Mercury's magnetotail. Discussion and Conclusions makeup the final two sections.

\section{Instrumentation and Data}

This study employs magnetic field and plasma measurements from MESSENGER. The ${ }_{87}$ magnetometer (MAG) measures magnetic field vector in a time resolution of 20 samples ${ }_{88}$ per second [Anderson et al., 2007]. The position data of MESSENGER were provided ${ }_{89}$ by accompanying with the magnetic field data at the same time resolution. The Fast 
90 Imaging Plasma Spectrometer (FIPS), which is one part of the Energetic Particle and

${ }_{91}$ Plasma Spectrometer (EPPS) [Andrews et al., 2007], measures ions with mass - amu ${ }_{92}$ over an energy range from $\sim 46 \mathrm{eV} / \mathrm{e}$ to $13.3 \mathrm{keV} / \mathrm{e}$ in every 10 seconds. However, 93 MESSENGER'S thermal sun shade limits its field of view to $\sim 1.4 \pi$ sr. FIPS also provides one minute proton moments, which were obtained by averaging the proton spectra over one minute intervals under the assumption of isotropic and subsonic of protons [Raines et al., 2011; Gershman et al., 2013].

The magnetic field data is in the Mercury Solar Magnetospheric (MSM) coordinate system, in which the $\mathrm{X}_{\mathrm{MSM}}$ axis is sunward, $\mathrm{Z}_{\mathrm{MSM}}$ axis points northward, and $\mathrm{Y}_{\mathrm{MSM}}$ axis completes the right-handed coordinate system. The center of MSM coordinate is $\sim 0.196 \mathrm{R}_{\mathrm{M}}$ northward offset from the Mercury's solid center [Alexeev et al., 2010; Anderson et al., 2010, 2011]. Position data of MESSENGER in X - Y plane were aberrated according to an angle between the anti-sunward solar wind and the orbital motion of Mercury around the Sun. The solar wind velocity was set to be constantly $-400 \mathrm{~km} / \mathrm{s}$ and orbital velocity of Mercury was daily averaged. The aberrated coordinate is labeled as $\mathrm{MSM}^{\prime}\left(\mathrm{X}_{\mathrm{MSM}}^{\prime}, \mathrm{Y}_{\mathrm{MSM}}^{\prime}, \mathrm{Z}_{\mathrm{MSM}}^{\prime}\right)$. The position aberration will not affect $\mathrm{Z}_{\mathrm{MSM}}$.

MESSENGER entered the orbit around Mercury on 11 March 2011, and impacted the surface of Mercury on 30 April 2015. The MESSENGER orbits could be divided into 'hot' and 'warm' seasons according to the locations of the periapsides [Slavin et al., 2014]. Hot seasons correspond to the orbits for which periapsis was located on the dayside and the warm seasons with them on the nightside. During the hot seasons, MESSENGER normally crossed the Mercury's magnetotail at a distance between $\sim-1.8 \mathrm{R}_{\mathrm{M}}$ and $-3 \mathrm{R}_{\mathrm{M}}$, ${ }_{112}$ which was close to the mean near Mercury neutral line (NMNL) [Slavin et al., 2012b; Poh 
${ }_{113}$ et al., 2017b]. This study surveys all of the hot seasons for the presence of flux ropes.

${ }_{114}$ Table 1 shows the start and end times for the 14 hot seasons between 23 March 2011

115 and 6 April 2015. The central plasma sheet was defined to by $\beta_{\mathrm{p}}>0.5$ [Sun et al., 2016],

${ }_{116}$ where the $\beta_{\mathrm{p}}$ is the ratio of proton thermal pressure to the magnetic pressure in the one minute data set, where the magnetic field data is averaged down to the same one minute intervals.

\section{Magnetotail Flux Rope Embedded in Current Sheet}

\subsection{A Case of Flux Rope}

A large amplitude flux rope was observed by MESSENGER between 03:12:45 and 03:12:55 UT on 17 May 2014 (Figure 1). The flux rope, marked by the shaded region, shows clearly bipolar signature in $B_{\mathrm{z}}$ which corresponds to peaks in $B_{\mathrm{y}}$ and $B_{\mathrm{t}}$. At $\sim$ 03:12:49 UT, $B_{\mathrm{y}}$ rapidly increased from $\sim 30 \mathrm{nT}$ to $\sim 94 \mathrm{nT}$ in less than one second and decreased to $\sim 30 \mathrm{nT}$ in the following second. Meanwhile, $B_{\mathrm{z}}$ exhibited a bipolar signature with an amplitude from peak to peak of $\sim 60 \mathrm{nT}$.

The magnetic field variation of this flux rope was revealed in the application of Minimum variance analysis (MVA) [Sonnerup and Cahill, 1967; Sonnerup and Scheible, 1998; Zong et al., 2003]. The results show that the maximum eigenvalue is close to the intermediate eigenvalue $\left(\lambda_{\max } / \lambda_{\text {int }} \sim 2\right)$, and both of the maximum and intermediate eigenvalues are much larger than the minimum eigenvalue $\left(\lambda_{\text {int }} / \lambda_{\min } \sim 48\right)$, which are the typical results for the application of MVA on flux rope. Figures 1a and 1b show the hodograms of the magnetic field of the flux rope under local coordinate determined by MVA. One hodogram is in $B_{\max }-B_{\text {int }}$ (Figure 1a), the other is in $B_{\max }-B_{\min }$ (Figure 1b). It shows that the 
${ }_{133}$ magnetic field rotates over $180^{\circ}$ in $B_{\max }-B_{\text {int }}$ while shows a straight line in $B_{\max }-B_{\min }$,

which further confirms the magnetic field variations of this flux rope.

\subsection{Selection Criteria for Flux Ropes}

This study applies the criteria in Sun et al. [2016] to select flux ropes in the plasma ${ }_{136}$ sheet at Mercury. In brief summary of the criteria, i) the $\Delta B_{\mathrm{t}}>10 n T\left(B_{\mathrm{t}}\right.$ enhancement) ${ }^{137}$ and $\Delta B_{\mathrm{z}}>15 n T\left(B_{\mathrm{z}}\right.$ bipolar change), ii) clear $B_{\mathrm{y}}$ enhancement, iii) clear magnetic field rotation in the MVA hodograms, and iv) events should be located inside the plasma sheet $\left(\beta_{\mathrm{p}}>0.5\right)$. Furthermore, this study has considered the plasma sheet durations under extreme solar wind conditions and includes plasma sheet crossings of 14 hot seasons.

We obtained 168 flux ropes in the 977 plasma sheet crossings among the 14 hot seasons, in which 135 are moving planetward and the other 33 events are moving tailward. Spatial distributions of the 168 flux ropes are shown in Figure 2 as blue crosses. Red lines are the orbits of MESSENGER during the hot season from 5 November 2011 to 1 December 2011, the first hot season in Table 1. The average magnetopause and bow shock locations of Mercury's magnetosphere obtained from Winslow et al. [2013] are shown in blue and green lines, respectively. In statistical, the mean increment of $B_{\mathrm{t}}$ of the 168 flux ropes is $\sim 17 \mathrm{nT}$, and is $\sim 77 \%$ in relative amplitude $\left(\Delta B_{\mathrm{t}} / B_{\mathrm{t}}\right)$. The distribution of flux ropes is skewed toward dawnside on the magnetotail, which is similar to the previous observations [Sun et al., 2016; Smith et al., 2017]. In this figure, 126 events were located on the dawnside $\left(\mathrm{Y}_{\mathrm{MSM}}^{\prime}<0\right)$, and 42 events were on the duskside $\left(\mathrm{Y}_{\mathrm{MSM}}^{\prime}>0\right)$. In the 977 plasma sheet crossings, 461 orbits were on the dawnside and 416 were on the duskside according to the intersections of orbits and magnetic equatorial plane. There was $\sim 10 \%$ more orbits on the dawnside than on the duskside, however, this should not account for 
${ }_{155}$ three times difference between the numbers of flux ropes on the dawnside and on the duskside.

\subsection{Force Balance of the Flux Ropes}

In this section, the force balance demonstrated by the magnetohydrostatic equation of the flux ropes in Mercury's magnetotail is examined. This equation is an equilibrium between plasma thermal pressure gradient force $(\nabla p)$ and Hall force $(\vec{J} \times \vec{B})$. The Hall force contains magnetic pressure gradient force $\left(\nabla \frac{B^{2}}{2 \mu_{0}}\right)$ and magnetic tension force $(\vec{B}$. $\left.\nabla \vec{B} / \mu_{0}\right)$. The magnetohydrostatic equation is an equalibrium between pressure gradient and magnetic tension, hereafter, we termed it as pressure-tension equilibrium equation. Along the normal direction $\left(N\right.$, mostly along $\left.\mathrm{Z}_{\mathrm{MSM}}\right)$ of the tail current sheet, the pressuretension equilibrium equation could be written as:

$$
\frac{\partial}{\partial N}\left(p+\frac{B^{2}}{2 \mu_{0}}\right)=\frac{B_{T}}{\mu_{0}} \frac{\partial B_{N}}{\partial T}
$$

, where $B_{N}$ is the normal magnetic field component (close to $B_{\mathrm{z}}$ ), $B_{T}$ is the tangential magnetic field component (close to $B_{\mathrm{x}}$ ), $p$ is the plasma thermal pressure. It is difficult to make a precise evaluation of this equation with only suitable magnetic field measurements, which is the case for MESSENGER observations. However, we can approximately estimate the force balance through the parameter differences between inside and outside of flux ropes on both sides of the equation [Paschmann et al., 1982]:

$$
\frac{\Delta\left(p+\frac{B^{2}}{2 \mu_{0}}\right)}{\Delta N}=\frac{B_{T}}{\mu_{0}} \frac{\left(B_{N^{+}}-B_{N^{-}}\right)}{\Delta T}
$$

${ }_{171}$. Here $B_{N^{ \pm}}$are the positive and negative extreme values inside the flux rope during 172 observation, and $B_{T}$ is taken as the total field adjacent to the flux rope (which is $\sim 31.0 \mathrm{nT}$ 
${ }_{173}$ for the case that shown in Figure 1). $\Delta T$ and $\Delta N$ denote the scale along the tangential

177

${ }^{191} \mathrm{X}_{\mathrm{MSM}}^{\prime}$ and $\left|B_{L}\right|$ was revealed in Mercury's tail:

$$
\left|B_{L}(\mathrm{X})\right|=A \cdot|\mathrm{X}|^{-D}+C
$$

${ }^{192}$, where $\left|B_{L}(\mathrm{X})\right|$ is the lobe field magnitude, $\mathrm{X}$ is the $\mathrm{X}_{\mathrm{MSM}}^{\prime}, A$ is the scaling constant, $D$ ${ }_{193}$ is the power law exponent, $C$ is the asymptotic magnetic field. Figure 3a shows the fit of 
13 14
D R A F T 


\subsection{Models of Flux Rope Embedded in Current Sheet}

The Models of flux rope embedded in current sheet applied in this study was developed by Kivelson and Khurana [1995], hereafter this model is referred to as KK95. This model was based on the periodic sheet pinch solution of the Ampère's law [Schindler et al., 1973]. A basic assumption of this model is that magnetic field and plasma thermal pressure show ${ }_{230}$ no gradient along the axial direction, which is approximately along the $\mathrm{Y}_{\mathrm{MSM}}^{\prime}$. The KK95 model includes a force-free model and a non-force-free model. The solution of force-free flux rope in consideration of the existence of $B_{\mathrm{y}}$ can be written as

$$
\left\{\begin{array}{l}
B_{\mathrm{x}}=\left(\frac{B_{L}}{\chi}\right) \sqrt{1+\varepsilon^{2}} \sinh \left(\frac{z}{L}\right) \\
B_{\mathrm{y}}=\left(\frac{B_{L}}{\chi}\right) \sqrt{1+\left(\frac{\chi B_{\mathrm{y}}}{B_{L}}\right)^{2}} \\
B_{\mathrm{z}}=\varepsilon\left(\frac{B_{L}}{\chi}\right) \sin \left(\frac{x}{L}\right)
\end{array}\right.
$$

where $B_{L}$ is the magnetic field strength in the lobe, $L$ is the thickness of the tail current sheet, $\varepsilon$ is the shape factor, $B_{\mathrm{y} 0}$ is the background $B_{\mathrm{y}}$, and $\chi$ is 


$$
\chi=\varepsilon \cos \left(\frac{x}{L}\right)+\sqrt{1+\varepsilon^{2}} \cosh \left(\frac{z}{L}\right)
$$

${ }_{235}$ In these equations, only $\varepsilon$ is a free parameter, and it determines the shape of the periodic ${ }_{236}$ sheet pinch. The larger the value of $\varepsilon$, the closer the shapes of magnetic field lines are ${ }^{237}$ circular. The $\varepsilon$ is obtained as a least squares fit result. However, when the thermal pressure gradient $(\nabla p)$ cannot be ignored, force balance equations in $X-Z$ plane should ${ }_{239}$ consider the contribution from thermal pressure gradient $(\vec{J} \times \vec{B}=\nabla p)$. In KK95 model, they consider the thermal pressure in the form of

$$
p(x, z)=\frac{p_{0}}{\chi^{2}}\left(1-\gamma \varepsilon / \chi^{\kappa-2}\right)
$$

where $p_{0}$ is the thermal pressure in the center of tail current sheet, and $\gamma$ and $\kappa$ are parameters determining the spatial profile of the pressure. The self-consistent solution for a non-force-free flux rope, after consideration of the above thermal pressure profile is given by:

$$
\left\{\begin{array}{l}
B_{\mathrm{x}}=\left(\frac{B_{L}}{\chi}\right) \sqrt{1+\varepsilon^{2}} \sinh \left(\frac{z}{L}\right) \\
B_{\mathrm{y}}=\left(\frac{B_{L}}{\chi}\right) \sqrt{\left(1-\frac{2 \mu_{0} p_{0}}{B_{L}{ }^{2}}\right)+\frac{2 \mu_{0} p_{0} \gamma \varepsilon}{B_{L}{ }^{2} \chi^{\kappa-2}}+\left(\frac{B_{\mathrm{y} 0} \chi}{B_{L}}\right)^{2}} \\
B_{\mathrm{z}}=\varepsilon\left(\frac{B_{L}}{\chi}\right) \sin \left(\frac{x}{L}\right)
\end{array}\right.
$$

In comparison with the Lundqvist solution based force-free flux rope model which solves the Bessel function [e.g., Lundquist, 1950; Burlaga, 1988; Lepping et al., 1996; Slavin et al., 2003], the KK95 non-force-free model takes into account not only the thermal pressure contribution, but also the boundary conditions. In this model, the variation of thermal pressure influences the spatial distribution of $B_{\mathrm{y}}$, but not $B_{\mathrm{x}}$ and $B_{\mathrm{z}}$. When $\varepsilon$ is close to 
${ }_{250}$ 0, equations (6) and (7) degenerate to the Harris Current Sheet (HCS) solution [Harris, 251 1962]:

$$
\left\{\begin{array}{l}
B_{\mathrm{x}}=B_{L} \tanh \left(\frac{z-z_{0}}{L}\right) \\
p=p_{0} \operatorname{sech}^{2}\left(\frac{z-z_{0}}{L}\right)
\end{array}\right.
$$

${ }^{252}$ Hence, when $z$ is far away $(>>L)$ from the center of flux rope, the magnetic field from the KK95 model is close to the values expected from the HCS model. Since the KK95 ${ }_{254}$ model relies on the basic parameters of the magnetotail current sheet, the thickness of the current sheet $(L)$ for instance, we have applied HCS model into the magnetic fields during the magnetotail crossing to obtain these parameters.

Figure 4 shows the plasma sheet crossing of MESSENGER during which the flux rope in ${ }_{258}$ Figure 1 was observed. In Figure 4, MESSENGER travelled from the northern hemisphere $\left(B_{\mathrm{x}}>0\right)$ to the southern hemisphere $\left(B_{\mathrm{x}}<0\right)$ and crossed the plasma sheet. The flux rope was observed near the central part of the plasma sheet, which is indicated by the dashed red line. HCS fitting only employs magnetic field measurements in the southern hemisphere to mitigate the effects from dipole magnetic field, since the MESSENGER is 263 closer to the planet in the northern hemisphere. The measured magnetic field has been transformed into the local coordinate system in the HCS fitting [Sun et al., 2017; Poh et al., 2017b; Rong et al., 2018]. Figure 4e shows the fitting result. The black line represents the measured magnetic field, and the red line is the HCS best fit. These two are coincident indicating a very good fit. The dashed blue line shows the thermal pressure distribution in this current sheet from the HCS fitting, and the blue dots are proton thermal pressure from one minute average moments of FIPS. The blue dots are much lower than the dashed 270 blue line, which could be due to, i) the one minute moments averaged over the peak values 
${ }^{271}$ of thermal pressure in the central of the current sheet (especially when there were few $287 \quad 4 \mathrm{e})$.

Since FIPS cannot resolve the background flow velocity for a single event due to the field of view limitation, we set the travelling speed of flux rope to be a free parameter to be determined by the best fit to the flux ropes, the Alfvén speed $\left(\frac{B_{L}}{\sqrt{\mu_{0} \mathrm{n}_{\mathrm{p}} \mathrm{m}_{\mathrm{p}}}}\right)$ estimated from the $B_{L}$, the lobe magnetic field, and $\mathrm{n}_{\mathrm{p}}$, proton density around flux ropes, was set as an upper limit. In the study of DiBraccio et al. [2015], they assumed a speed of $465 \mathrm{~km} / \mathrm{s}$ for ${ }_{293}$ all of the flux ropes, which was obtained by averaging over the local Alfvén speeds for all 
${ }_{294}$ adjacent plasma sheets. In this fitting, we apply $x=v\left(t-t_{0}\right) \cos \theta$ and $z=v\left(t-t_{0}\right) \sin \theta+\Delta z$

\section{MESSENGER's position and the $z_{0}$ resulted from the HCS modelling.}

The Alfvén speed $\left(\frac{B_{L}}{\sqrt{\mu_{0} \mathrm{n}_{\mathrm{p}} \mathrm{m}_{\mathrm{p}}}}\right)$ for the flux rope in Figure 1 is determined to be $\sim 910 \mathrm{~km} / \mathrm{s}$.

${ }_{300}$ Together with the parameters of current sheet, the fitting results of the flux rope were shown in Figures 1c to 1f as the dashed red lines. The similarity between observation and model fields indicates a good fitting. The fitting suggested that the flux rope had a so3 travelling speed of $\sim 900 \mathrm{~km} / \mathrm{s}$, magnetic flux content of $\sim 0.010 \mathrm{MWb}$, semi-major axis (scale along $\mathrm{X}_{\mathrm{MSM}}^{\prime}$ ) of $\sim 600 \mathrm{~km}, \varepsilon$ of $0.56, \gamma$ of $0.2, \kappa$ of 5 . The magnetic flux content of flux rope was obtained by integrating $B_{\mathrm{y}}$ in the cross section inside the outmost field line, i.e., $\Phi=\iint B_{\mathrm{y}} \mathrm{d} x \mathrm{~d} z$. Figure 5 shows the two-dimensional distributions of $B_{\mathrm{y}}$ and $p$ in the plane transverse to the axis of this flux rope from the KK95 model. MESSENGER crossed close to the center axis of this flux rope. The $B_{\mathrm{y}}$ in the center was around minimum in the central part of the flux rope. While, the thermal pressure inside the flux rope is significantly larger than the ambient thermal pressure. The results revealed that the scale of flux rope was around twice the the scale along the x-axis from DiBraccio et al. [2015] and Smith et al. [2017] whose force free model assumes a circular cross section. The core field and magnetic flux content were also much larger than the average values from their studies. To further evaluate the result from a single case study, a statistical analysis ${ }_{316}$ on the flux rope properties determined using the KK95 model is presented below. 


\section{Statistical Results on the Flux Ropes}

326 et al., 2015]

$$
\chi^{2}=\frac{\sum_{i=1}^{N} \sum_{j=x, y, z}\left[\left(B_{j o}(i)-B_{j m}(i)\right) / B_{t o}(i)\right]^{2}}{N}
$$

, where $B_{x o}, B_{y o}, B_{z o}$, and $B_{t o}$ are the components and magnitude of the measured magnetic fields, and $B_{x m}, B_{y m}$, and $B_{z m}$ are the components from the KK95 model. $N$ 329 is the number of data points. The parameters of the model corresponding to the smallest $\chi^{2}$ were output. After obtaining the $\chi^{2}$ of the 103 flux ropes, a threshold of $\chi^{2}<0.1$ to further select the events results in 28 events. A different threshold of $\chi^{2}<0.05$ gives 20 events. The statistical results of the 28 and 20 flux ropes were summarized in Figure 6 . The distributions from the two threshold of $\chi^{2}<0.1$ (white bars) and $\chi^{2}<0.05$ (grey bars) are similar and result in similar values. In the next paragraph, we will discuss the results from $\chi^{2}<0.1$ (white bars). 
52 for the 20 flux ropes in Figure 6. Force free results can be found in the supplementary material as Figure S1. The statistical results from force-free model give a mean flux content of $\sim 0.012 \mathrm{MWb}$, which is $\sim 35 \%$ smaller than the value from non-force-free model. This indicates that the non-force-free model did output a relatively higher flux content for flux ropes. The mean core field is $\sim 60 n T$ from the force-free model, which is similar to the values $\left(\sim 69.1 n T\right.$ for $\chi^{2}<0.05, \sim 57.5 n T$ for $\chi^{2}<0.1$. $)$ from non-force${ }_{358}$ free model. While the radius is $\sim 367 \mathrm{~km}$ from the force-free model, corresponding to a 
${ }_{359}$ cross-sectional area of $\sim 5.4 \times 10^{5} \mathrm{~km}^{2}$. The mean cross-sectional area is $\sim 9.2 \times 10^{5} \mathrm{~km}^{2}$

Figure 6e shows that the semi-minor (along $\mathrm{Z}_{\mathrm{MSM}}^{\prime}$ ) is $\sim 356 \mathrm{~km}$. On one hand, the scales are much larger than the scales in previous studies (454 km in DiBraccio et al. [2015] and $262 \mathrm{~km}$ Smith et al. [2017]). On the other hand, semi-major is much larger than the semiminor indicating that flux ropes are flattened along the $\mathrm{X}_{\mathrm{MSM}}^{\prime}$, which consists with the flatten conclusion reached by Figure 3b. Plasma sheet density in Mercury's magnetotail plasma sheet is found to be $\sim 1$ to $10 \mathrm{~cm}^{-3}$ [Gershman et al., 2014; Sun et al., 2018; Poh et al., 2018], corresponding to ion inertial length of 80 to $230 \mathrm{~km}$. The scales of flux ropes resulted in KK95 model are several times the ion inertial length. In Figure 3b, the ratio between of average scale of flux rope along $\mathrm{Z}_{\mathrm{MSM}}^{\prime}$ and $\mathrm{X}_{\mathrm{MSM}}^{\prime}$ was estimated to be $\sim 0.66$. The model in this study gives a ratio of $\sim 0.41$. One must note that the spacecraft did not always cross the center axis of the flux rope. Hence, the scale estimated from Figure 374 difference between the two values. Figure $6 \mathrm{f}$ shows the distribution of travelling speeds of the flux ropes. As noted earlier, we have employed a different way than DiBraccio et al. [2015] in determining the travelling speeds of flux ropes. The mean and median speeds are $\sim 560 \mathrm{~km} / \mathrm{s}$ and $535 \mathrm{~km} / \mathrm{s}$, respectively, which are slightly larger than $465 \mathrm{~km} / \mathrm{s}$ in DiBraccio et al. [2015]. 


\section{Discussions}


${ }_{424}$ guide field as proposed in the simulations [e.g., Nakamura et al., 2016]. This factor might

explain their poor correlation between core field of flux ropes and guide field.

\section{Conclusions}

This study has investigated the features of flux ropes in Mercury's magnetotail plasma sheet, including the force balance and flux rope structures. The spatial distribution of flux ropes shows clearly dawn-dusk asymmetry with more events being observed on the dawnside than on the duskside, which consists with the previous results [Sun et al., 2016]. An investigation on the force balance of flux ropes reveals that the magnetic pressure gradient force cannot be solely balanced by magnetic tension force in most of the flux ropes, implying the importance of thermal pressure inside the flux ropes. By employing a non-force-free flux rope model, the thermal pressure differences, core field, scales, and flux contents were investigated. The mean value of the largest thermal pressure differences along $\mathrm{X}_{\mathrm{MSM}}^{\prime}$ of the flux ropes was $\sim 1.40 \mathrm{nPa}$. The average core field was estimated to be $\sim 57.5 \mathrm{nT}$, and flux content was $\sim 0.019 \mathrm{MWb}$. The average core field corresponds ${ }^{437}$ to a similar value of pressure, i.e. $\sim 1.31 \mathrm{nPa}$, as the largest thermal pressure differences ${ }^{438}$ along $\mathrm{X}_{\mathrm{MSM}}^{\prime}$. The flux ropes had a flattened structure with scale in the $\mathrm{X}_{\mathrm{MSM}}^{\prime}$ direction $(\sim 851 \mathrm{~km})$ being larger than in the $\mathrm{Z}_{\mathrm{MSM}}^{\prime}(\sim 333 \mathrm{~km})$. The scales of the flux ropes were several times the background proton inertial length. Besides, the average travelling speed of flux ropes was estimated to be $\sim 560 \mathrm{~km} / \mathrm{s}$.

Compare with the results obtained from force-free model of flux ropes in Mercury's ${ }_{443}$ magnetotail [DiBraccio et al., 2015; Smith et al., 2017], the core field and flux content ${ }_{444}$ in this study were much larger than the previous results, in which the core field was ${ }_{445} \sim 22 \mathrm{nT}$ and flux content was $\sim 0.002 \mathrm{MWb}$. The scale of the flux rope in this study 
${ }_{446}$ was much larger than the previous value $(\sim 262 \mathrm{~km})$, but the average travelling speed was comparable $(465 \mathrm{~km} / \mathrm{s})$ [DiBraccio et al., 2015]. The magnetic flux contained by a

${ }_{448}$ flux rope in previous study was an order of magnitude smaller than the the magnetic flux

${ }_{449}$ carried by a DFB [Dewey et al., 2018], while this study reveals that the flux content of a flux rope is about one third of the flux of a DFB. It needs to note that Fear et al. [2017] argued that the amount of flux reconnected in the formation of the flux ropes could be ${ }_{452}$ greater than the flux rope contents, which might be more directly comparable with the DFB flux.

The importance of thermal pressure gradient in the force-balance of the flux ropes and ${ }^{455}$ the flatten structure indicate that the flux ropes observed by MESSENGER in Mercury's tail have only recently formed. The flux ropes still contained enough plasma to affect their magnetic structures as observed in PIC simulations of flux rope formation in thin current sheets [Chen et al., 2017]. The core field of the early stage flux rope could be influenced by the reconnecting magnetic field, which explained the weak correlation between core field of flux ropes and the guide field as shown in previous studies [Smith et al., 2017; ${ }_{461} \quad$ Ding and Rong, 2018].

Acknowledgments. This work is supported by the National Natural Science Foundation of China (grants 41421003, 41704163, and 41627805). The University of Michigan contributions were supported by NASA's Solar System Workings program through grant NNX15AH28G.. MESSENGER data used in this study were available from the Planetary Data System (PDS): http://pds.jpl.nasa.gov. The MESSENGER project is supported by the NASA Discovery Program under contracts NASW-00002 to the Carnegie Institu${ }_{468}$ tion of Washington and NAS5-97271 to The Johns Hopkins University Applied Physics 
${ }_{469}$ Laboratory. We are grateful to MESSENGER Magnetometer and Fast Imaging Plasma

470 Spectrometer (FIPS) for providing the data. 


\section{References} messenger spacecraft, Space Science Reviews, 131(1), 523-556, doi:10.1007/s11214-007$9272-5$ 
Paschmann, G., G. Haerendel, I. Papamastorakis, N. Sckopke, S. J. Bame, J. T. Gosling, and C. T. Russell (1982), Plasma and magnetic field characteristics of magnetic flux transfer events, Journal of Geophysical Research: Space Physics, 87(A4), 2159-2168, doi:10.1029/JA087iA04p02159. 
Poh, G., J. A. Slavin, X. Jia, J. M. Raines, S. M. Imber, W.-J. Sun, D. J. Gershman, G. A. DiBraccio, K. J. Genestreti, and A. W. Smith (2017a), Coupling between mercury and its nightside magnetosphere: Cross-tail current sheet asymmetry and substorm current wedge formation, Journal of Geophysical Research: Space Physics, 122(8), 8419-8433, doi:10.1002/2017JA024266.

Poh, G., J. A. Slavin, X. Jia, J. M. Raines, S. M. Imber, W.-J. Sun, D. J. Gershman, G. A. DiBraccio, K. J. Genestreti, and A. W. Smith (2017b), Mercury's cross-tail current sheet: Structure, x-line location and stress balance, Geophysical Research Letters, $44(2)$, 678-686, doi:10.1002/2016GL071612.

Poh, G., J. A. Slavin, X. Jia, W.-J. Sun, J. M. Raines, S. M. Imber, G. A. DiBraccio, and D. J. Gershman (2018), Transport of mass and energy in mercury's plasma sheet, Geophysical Research Letters, 45(22), 12,163-12,170, doi:10.1029/2018GL080601.

Raines, J. M., J. A. Slavin, T. H. Zurbuchen, G. Gloeckler, B. J. Anderson, D. N. Baker, H. Korth, S. M. Krimigis, and R. L. McNutt (2011), Messenger observations of the plasma environment near mercury, Planetary and Space Science, 59(15), 2004 - 2015, doi:https://doi.org/10.1016/j.pss.2011.02.004, mercury after the MESSENGER flybys.

Raines, J. M., D. J. Gershman, T. H. Zurbuchen, M. Sarantos, J. A. Slavin, J. A. Gilbert, H. Korth, B. J. Anderson, G. Gloeckler, S. M. Krimigis, D. N. Baker, R. L. McNutt, and S. C. Solomon (2013), Distribution and compositional variations of plasma ions in mercury's space environment: The first three mercury years of messenger observations, Journal of Geophysical Research: Space Physics, 118(4), 1604-1619, doi: 10.1029/2012JA018073. 
Solomon, S. C., R. L. McNutt, R. E. Gold, M. H. Acuña, D. N. Baker, W. V. Boynton, C. R. Chapman, A. F. Cheng, G. Gloeckler, J. W. H. III, S. M. Krimigis, W. E. McClintock, S. L. Murchie, S. J. Peale, R. J. Phillips, M. S. Robinson, J. A. Slavin, D. E. Smith, R. G. Strom, J. I. Trombka, and M. T. Zuber (2001), The messenger mission to mercury: scientific objectives and implementation, Planetary and Space Science, $49(14)$, 1445 - 1465, doi:https://doi.org/10.1016/S0032-0633(01)00085-X, returns to Mercury. 


\section{Z11}

712

Zhao, C., C. T. Russell, R. J. Strangeway, S. M. Petrinec, W. R. Paterson, M. Zhou, B. J. Anderson, W. Baumjohann, K. R. Bromund, M. Chutter, D. Fischer, G. Le, R. Nakamura, F. Plaschke, J. A. Slavin, R. B. Torbert, and H. Y. Wei (2016), Force balance at the magnetopause determined with mms: Application to flux transfer events, Geophysical Research Letters, 43(23), 11,941-11,947, doi:10.1002/2016GL071568. and X. H. Han (2015), Mercury's three-dimensional asymmetric magnetopause, Journal of Geophysical Research: Space Physics, 120(9), 7658-7671, doi:10.1002/2015JA021425. Zhong, J., Y. Wei, Z. Y. Pu, X. G. Wang, W. X. Wan, J. A. Slavin, X. Cao, J. M. Raines, H. Zhang, C. J. Xiao, A. M. Du, R. S. Wang, R. M. Dewey, L. H. Chai, Z. J. Rong, and Y. Li (2018), Messenger observations of rapid and impulsive magnetic reconnection in mercury's magnetotail, The Astrophysical Journal Letters, 860(2), L20. 
Zong, Q.-G., B. Wilken, G. D. Reeves, I. A. Daglis, T. Doke, T. Iyemori, S. Livi, K. Maezawa, T. Mukai, S. Kokubun, Z.-Y. Pu, S. Ullaland, J. Woch, R. Lepping, and T. Yamamoto (1997), Geotail observations of energetic ion species and magnetic field in plasmoid-like structures in the course of an isolated substorm event, Journal of Geophysical Research: Space Physics, 102(A6), 11,409-11,428, doi:10.1029/97JA00076. Zong, Q.-G., T. Fritz, H. Spence, M. Dunlop, Z. Pu, A. Korth, P. Daly, A. Balogh, and H. Reme (2003), Bursty energetic electrons confined in flux ropes in the cusp region, Planetary and Space Science, 51(12), 821 - 830, doi:https://doi.org/10.1016/S00320633(03)00116-8, key Problems in Space Physics: Thin Magnetospheric Boundaries.

Zong, Q.-G., T. A. Fritz, Z. Y. Pu, S. Y. Fu, D. N. Baker, H. Zhang, A. T. Lui, I. Vogiatzis, K.-H. Glassmeier, A. Korth, P. W. Daly, A. Balogh, and H. Reme (2004), Cluster observations of earthward flowing plasmoid in the tail, Geophysical Research Letters, 31 (18), doi:10.1029/2004GL020692. 
Table 1: The start and end times of the MESSENGER hot seasons

\begin{tabular}{cccc}
\hline$\#$ & year & start doy & end doy \\
\hline 1 & 2011 & 309 & 335 \\
2 & 2012 & 33 & 58 \\
3 & 2012 & 122 & 144 \\
4 & 2012 & 210 & 232 \\
5 & 2012 & 297 & 318 \\
6 & 2013 & 64 & 79 \\
7 & 2013 & 146 & 163 \\
8 & 2013 & 231 & 254 \\
9 & 2013 & 318 & 342 \\
10 & 2014 & 43 & 65 \\
11 & 2014 & 132 & 151 \\
12 & 2014 & 218 & 238 \\
13 & 2014 & 305 & 328 \\
14 & 2015 & 27 & 52 \\
\hline
\end{tabular}

a doy, day of the year 
Flux Rope-2014-05-17
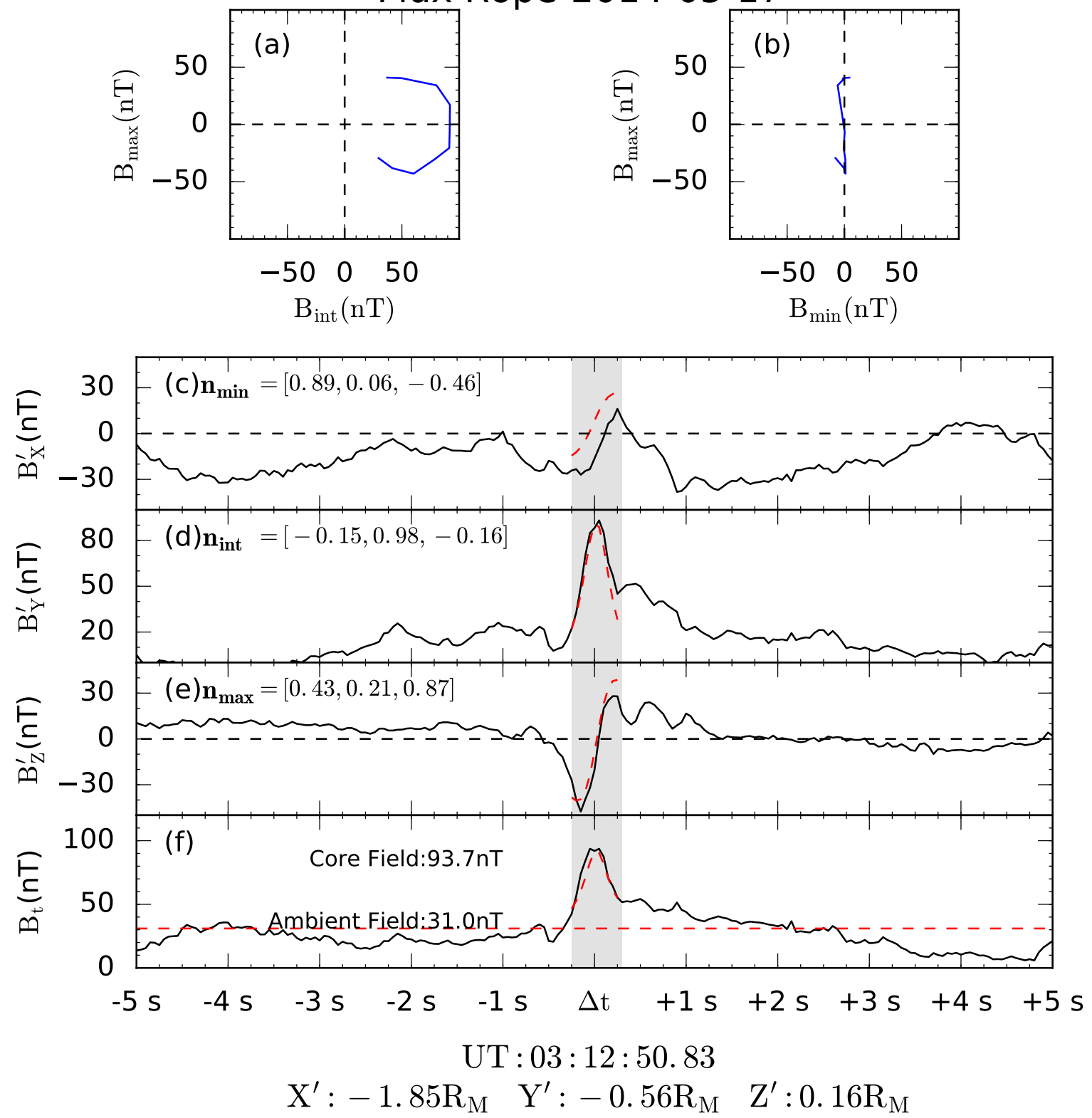

Figure 1: A flux rope case in Mercury's magnetotail at 03:12:50.83 UT, 17 May 2014. MVA magnetic field hodograms of the flux rope are shown in the planes of $B_{\max }-B_{\text {int }}$ (a) and $B_{\max }$ $B_{\text {min }}$ (b), respectively. (c) magnetic field x component, $B_{\mathrm{x}}$, (d) $B_{\mathrm{y}}$, (e) $B_{\mathrm{z}}$, (f) $B_{\mathrm{t}}$. Dashed red curves in the shaded region from (c) to (f) are the magnetic field from the fitting of KK95 model. 

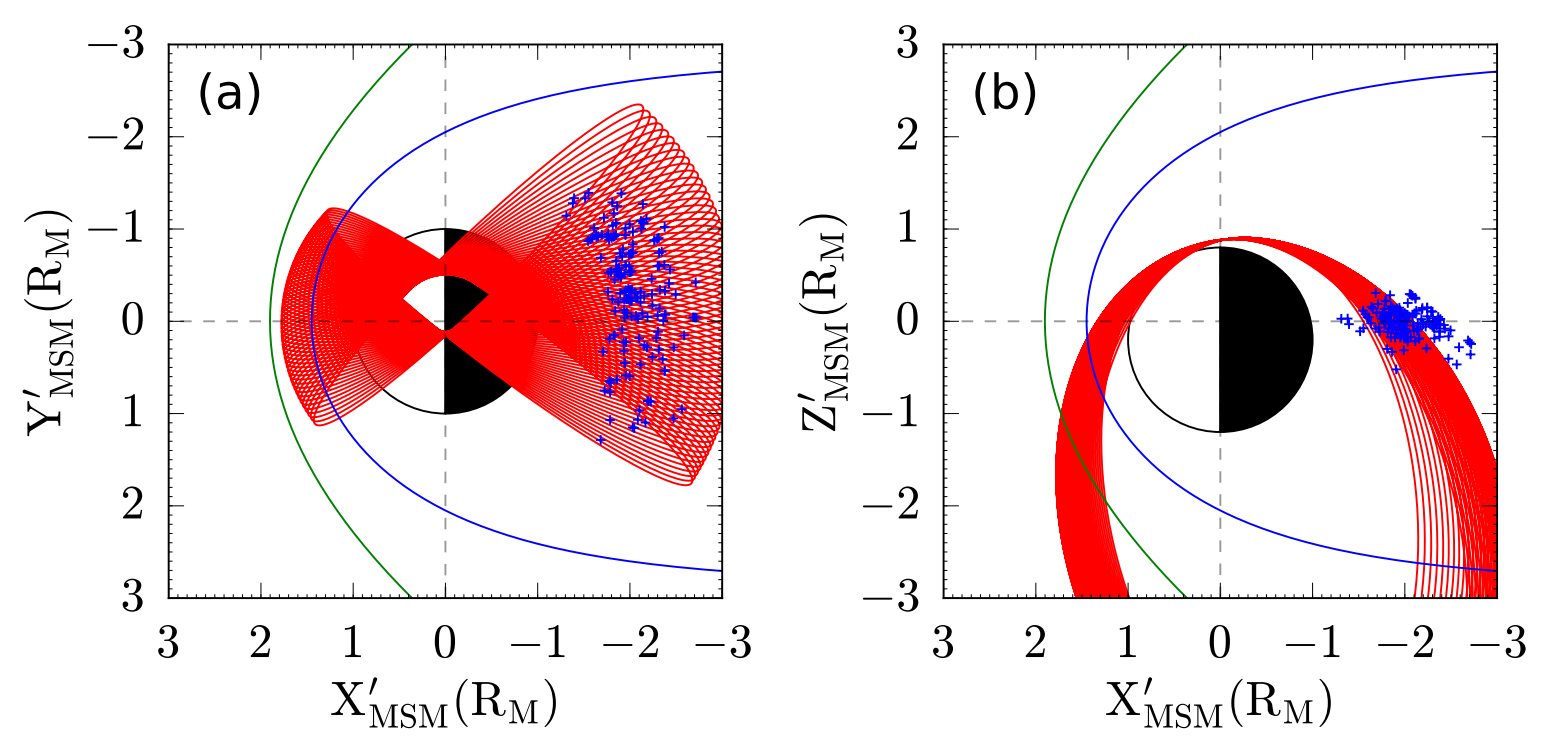

Figure 2: Spatial distributions of the 168 flux ropes in $\mathrm{X}_{\mathrm{MSM}}^{\prime}-\mathrm{Y}_{\mathrm{MSM}}^{\prime}(\mathrm{a})$ and $\mathrm{X}_{\mathrm{MSM}}^{\prime}-\mathrm{Z}_{\mathrm{MSM}}^{\prime}(\mathrm{b})$ planes, respectively. Blue crosses represent the flux ropes. MESSENGER orbits in the hot season from 2011-309 to 2011-335 are shown as red lines. The blue and green lines indicate the average locations of magnetopause and bow shock of Mercury's magnetosphere from [Winslow et al., 2013]. 

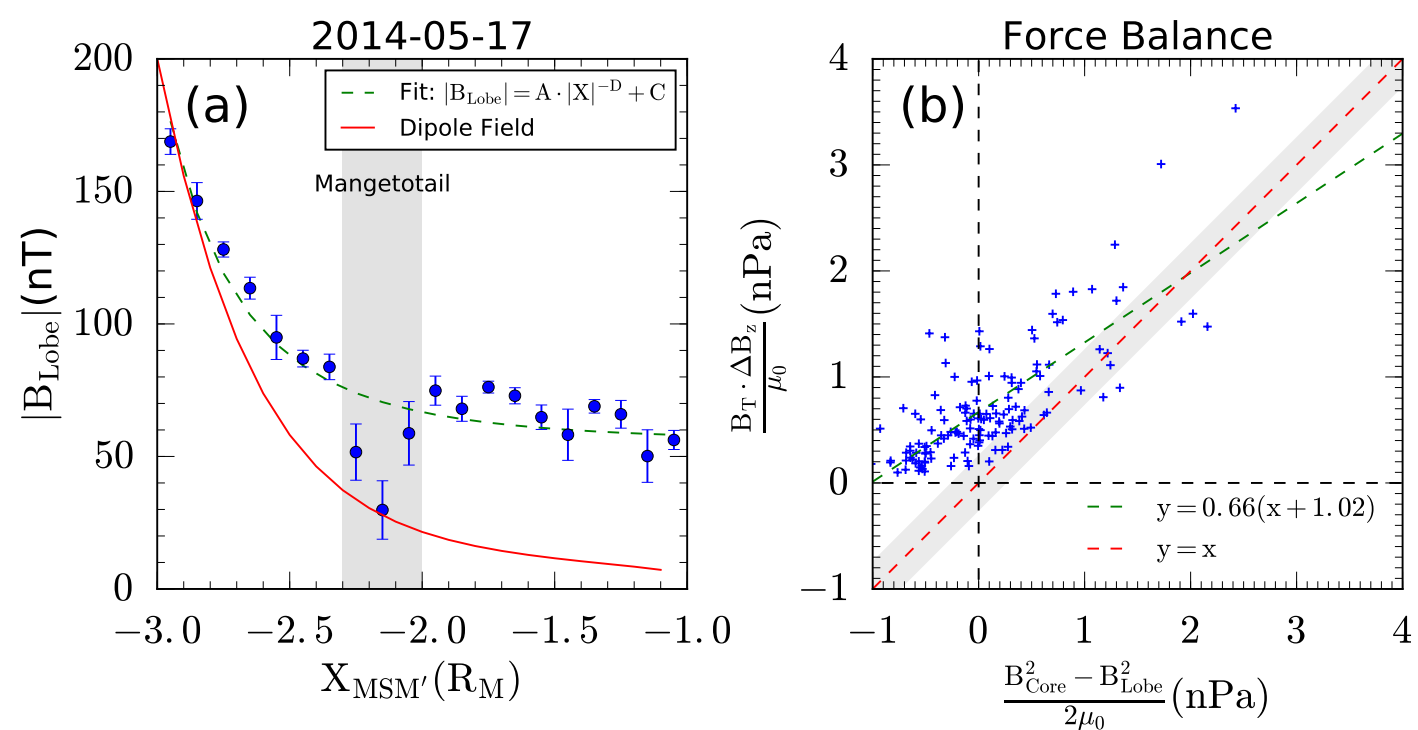

Figure 3: (a) Power Law fitting of the magnetic field intensity $\left(B_{\mathrm{t}}\right)$ along the tail distance $\left(\mathrm{X}_{\mathrm{MSM}}^{\prime}\right)$ for the first magnetotail passes on 17 May 2014. The red line indicates the magnitude of the dipole magnetic field of Mercury. The blue dots with error bars represent the intensities of the measured magnetic field, which are averaged over each $0.1 \mathrm{R}_{\mathrm{M}}$ bin (error bars here are the standard deviation). The dashed green line shows the power law fitting of the blue dots with the parameters $\mathrm{A}=144.8 \mathrm{nT}, \mathrm{D}=3.7$ and $\mathrm{C}=55.5 \mathrm{nT}$. (b) The distribution of magnetic pressure differences and magnetic tension force for the 143 flux ropes. Each cross indicates an event. The dashed red line has a slope of one. The dashed green line is the linear fit of the data points. The shaded region corresponding to the quasi-force-free criterion. 

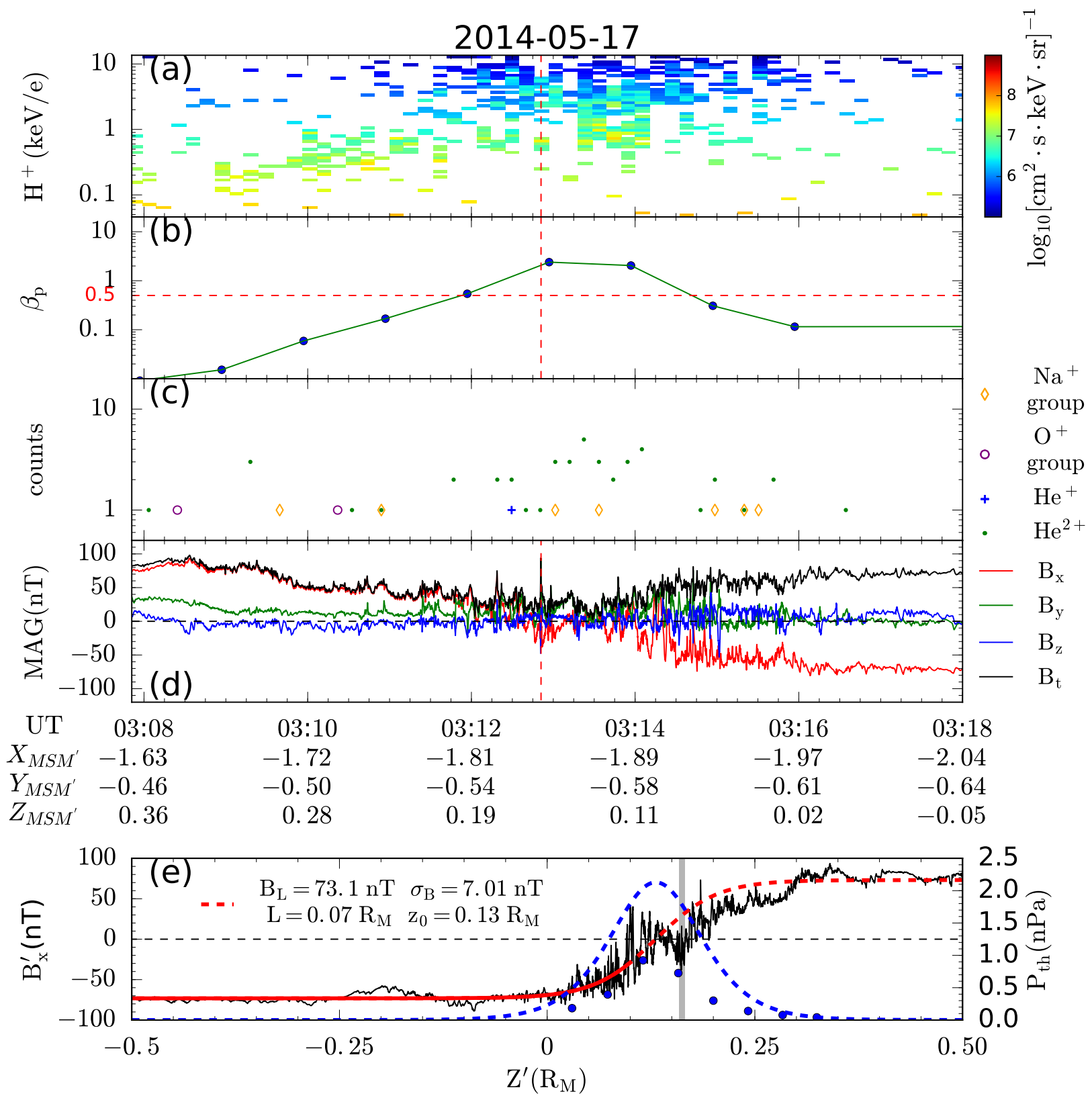

Figure 4: Plasma and magnetic field measurements from MESSENGER between 03:08 to 03:18

UT on 17 May 2014. (a) energy spectrum for proton differential particle flux, (b) plasma $\beta$ from one minute average proton moments, (c) heavy ion counts of four composition types, $\mathrm{He}^{+}$ (cross), $\mathrm{He}^{++}$(dots), $\mathrm{O}^{+}$group, $\mathrm{m} / \mathrm{q}=14-20$, (circle), and $\mathrm{Na}^{+}$group, $\mathrm{m} / \mathrm{q}=20-30$, (diamond), (d) magnetic field components, $B_{\mathrm{x}}$ (red), $B_{\mathrm{y}}$ (green), $B_{\mathrm{z}}$ (blue), $B_{\mathrm{t}}$ (black), (e) $B_{\mathrm{x}}^{\prime}$ measurements in local coordinate (black) and the fitting from Harris current sheet model (red), thermal pressure from Harris current sheet fitting (dashed blue line), thermal pressure from one minute proton moments (blue dots). 

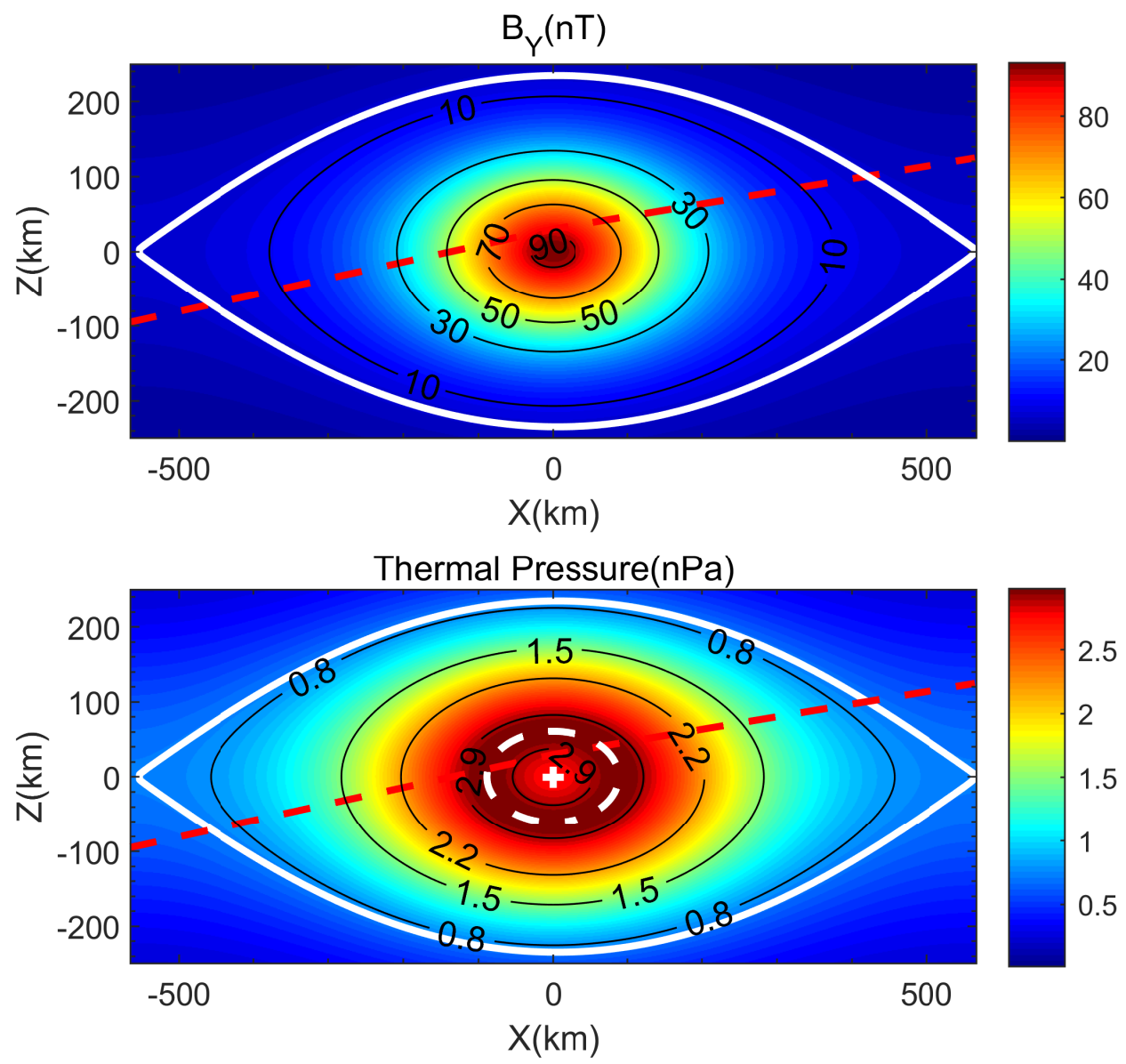

Figure 5: The core field, $B_{\mathrm{y}}$, (a) and thermal pressure, $p$, (b) distributions from the KK95 model for the flux rope in Figure 1. The dashed red line represents the trajectory of the spacecraft. Solid white line marks the boundary of the flux rope. Dashed white line and cross indicates the contour of peak $(p=2.98 \mathrm{nPa})$ and the central dip $(p=2.75 \mathrm{nPa})$ of thermal pressure in the flux rope. Black lines with values are the contour of $B_{\mathrm{y}}$ and $p$. 

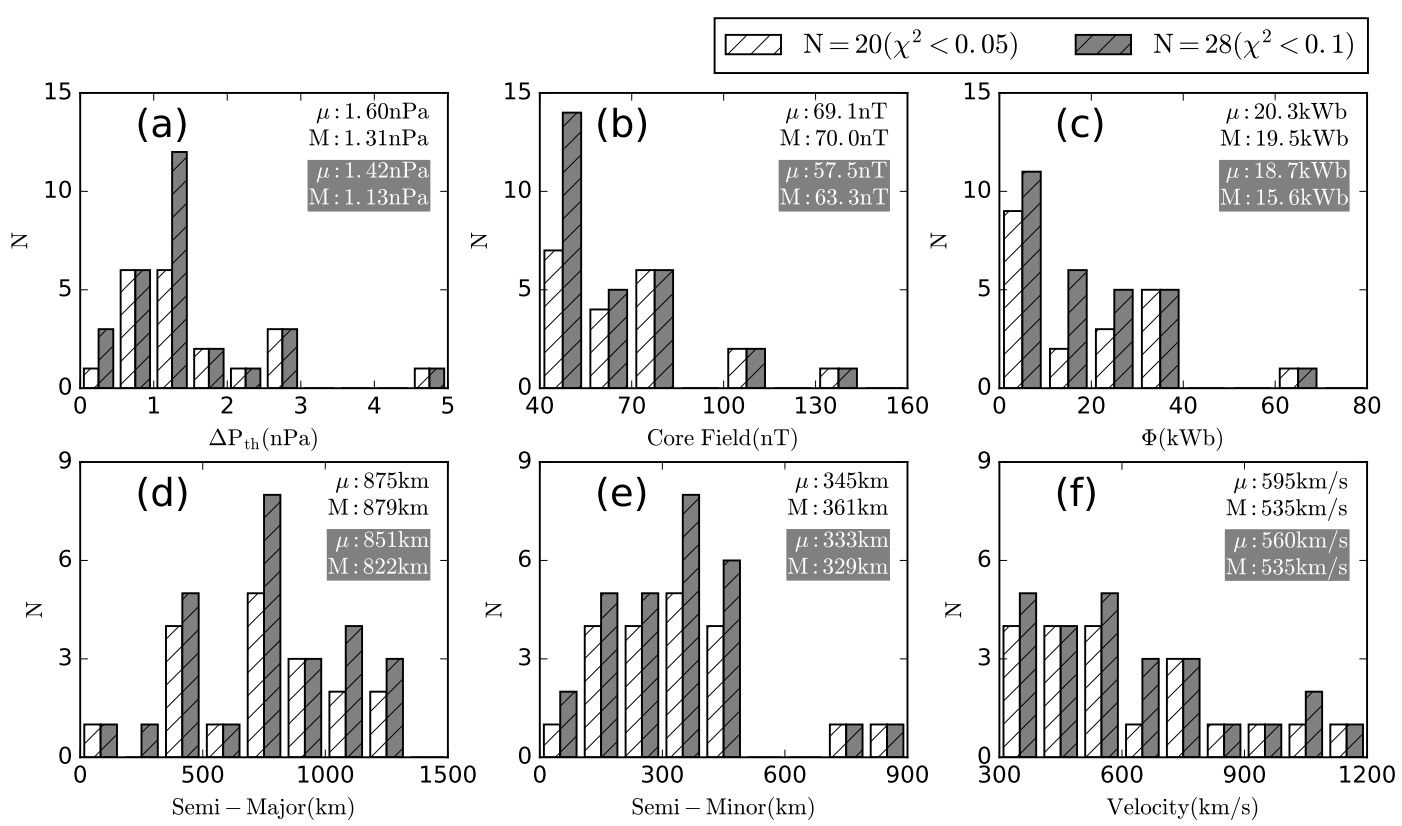

Figure 6: Statistical properties of the flux ropes resulted from the KK95 model. (a) The largest thermal pressure $(p)$ difference along the major axes, (b) the core field in the center of flux rope, (c) magnetic flux content, (d) semi-major axes (scale in $\mathrm{X}_{\mathrm{MSM}}^{\prime}$ ), (e) semi-minor axes (scale in $\mathrm{Z}_{\mathrm{MSM}}^{\prime}$ ), (f) The traveling speeds. The grey and white bars represent the distributions of event with $\chi^{2}<0.1$ and $\chi^{2}<0.05$, respectively. In each figure, $\mu$ represent the mean values. $M$ represent the median values. 
(a)

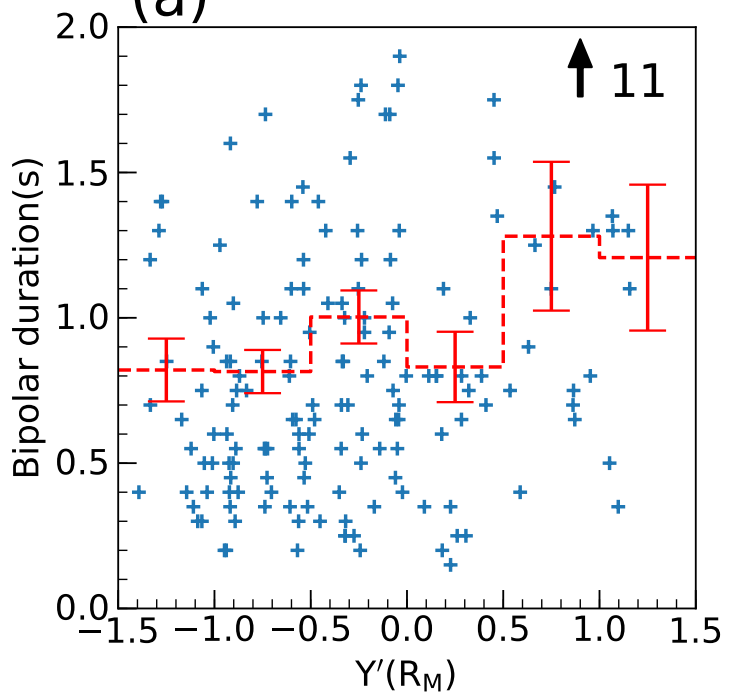

(b)

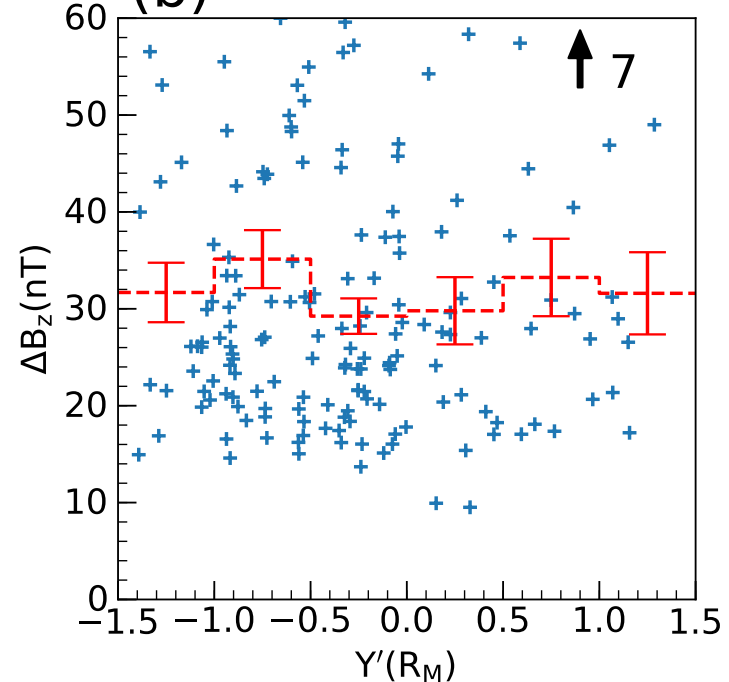

Figure 7: The distributions of the duration and amplitudes of flux ropes along $\mathrm{Y}_{\mathrm{MSM}}^{\prime}$. (a) the duration were obtained from peak to peak of $B_{\mathrm{z}}$. (b) the amplitude of $B_{\mathrm{z}}$ from peak to peak. Errorbars represent the standard error of the mean in each bin. Number of off-axis events is marked on the top right corner.Another version of this Figure with a wider range in $\mathrm{Y}$-axis is attached in supplementary material as Figure S2. 
Flux Rope-2014-05-17
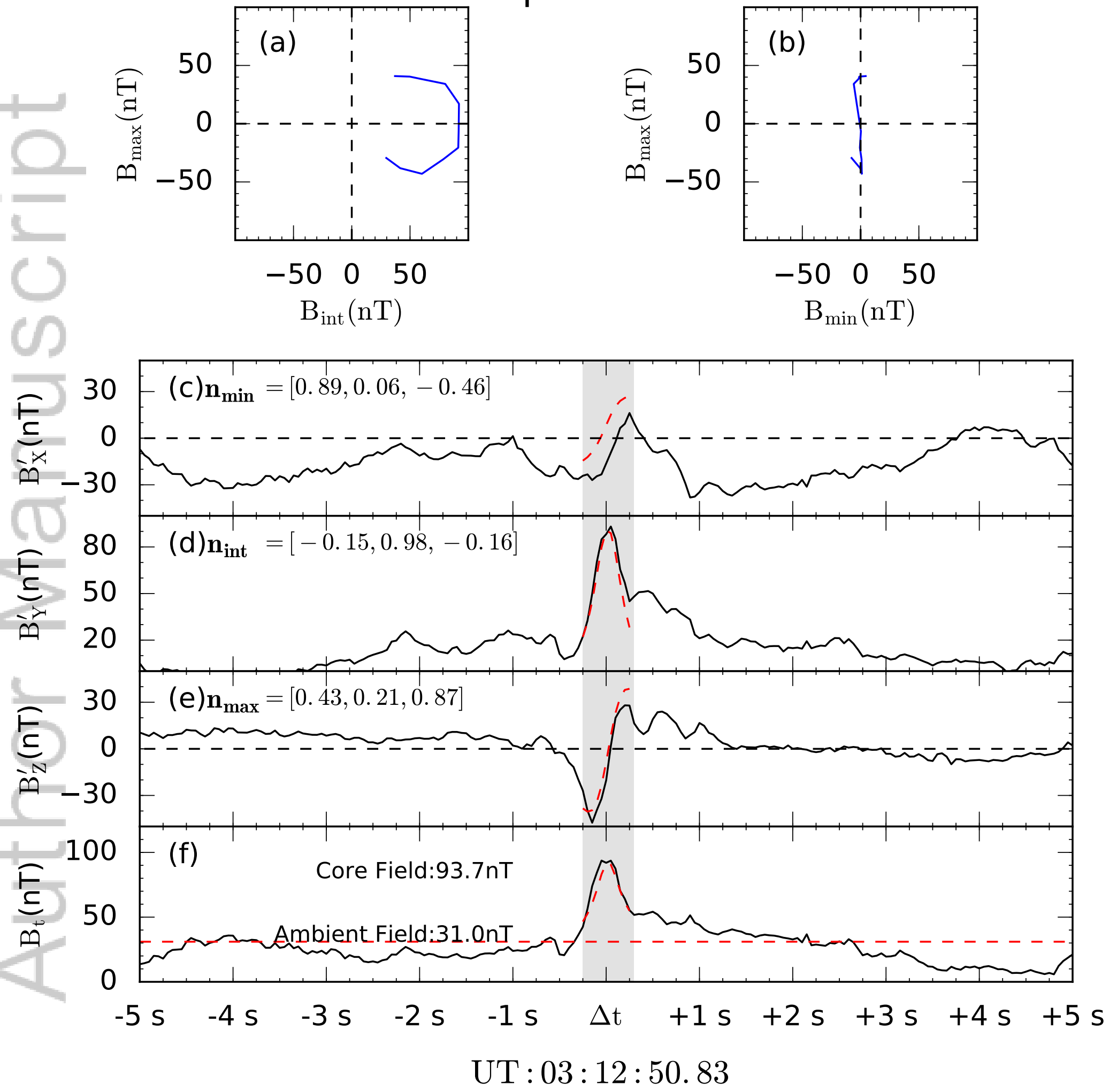

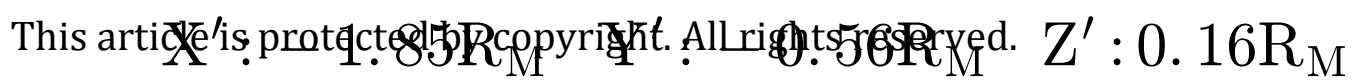




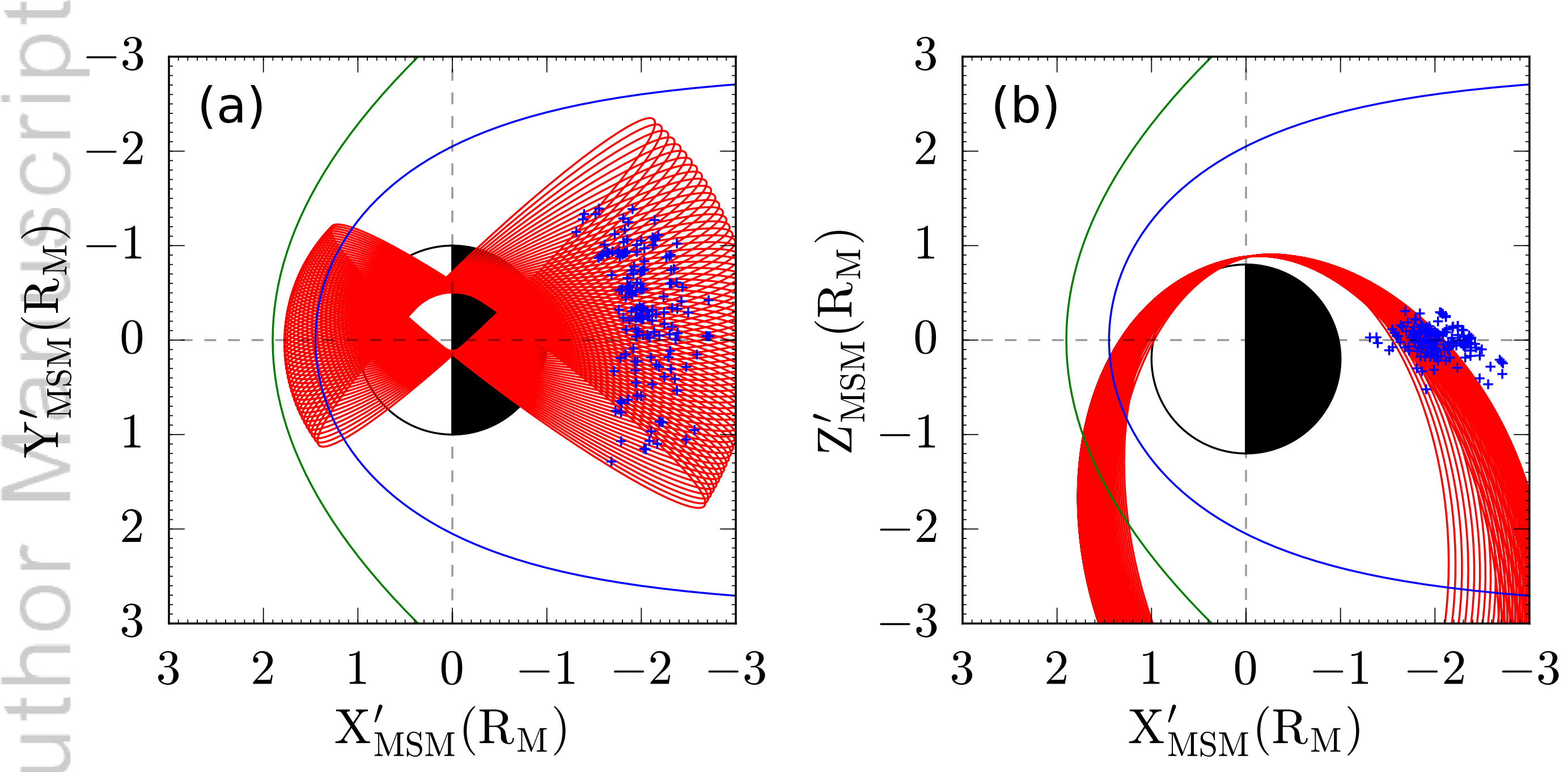

This article is protected by copyright. All rights reserved. 

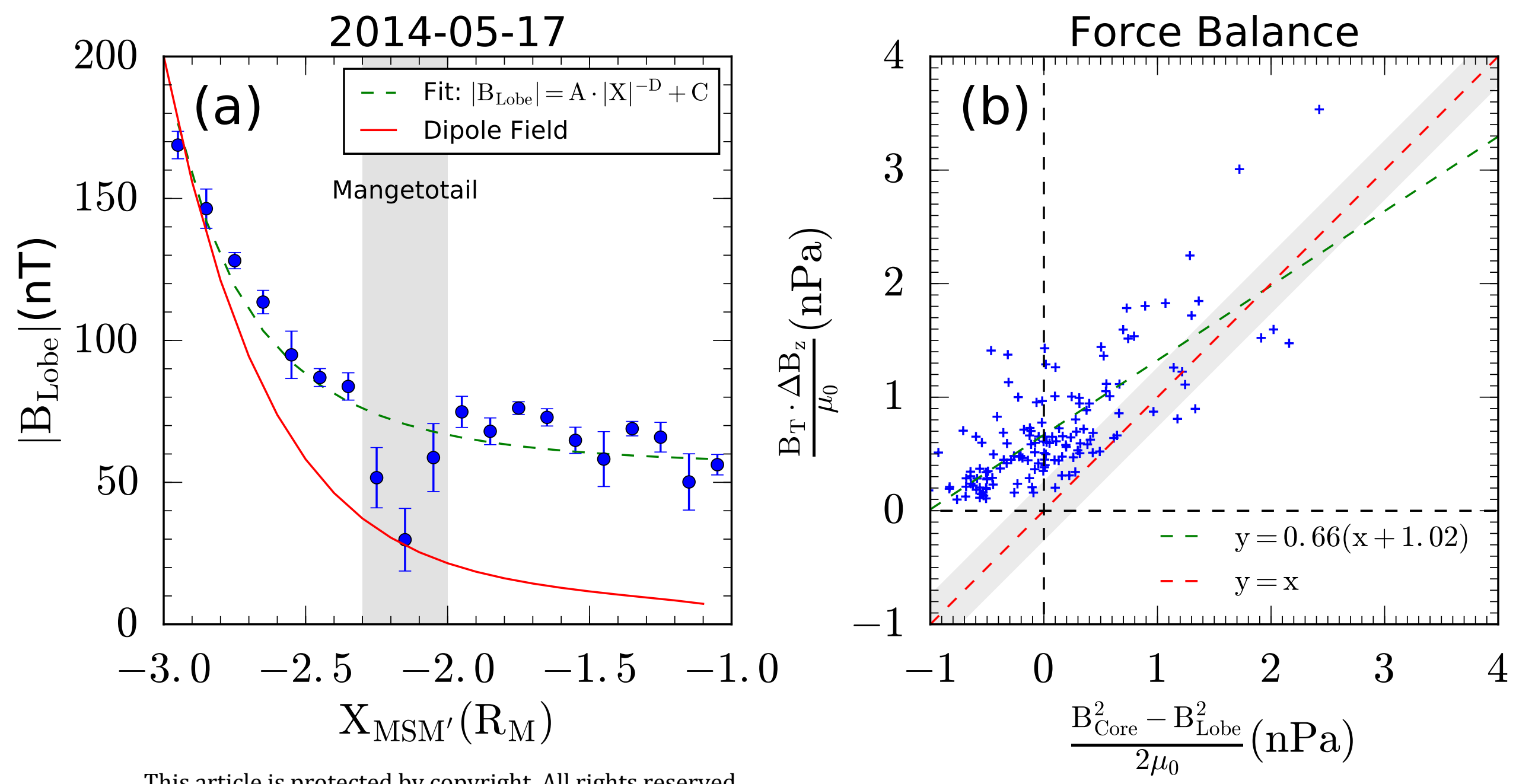

This article is protected by copyright. All rights reserved. 


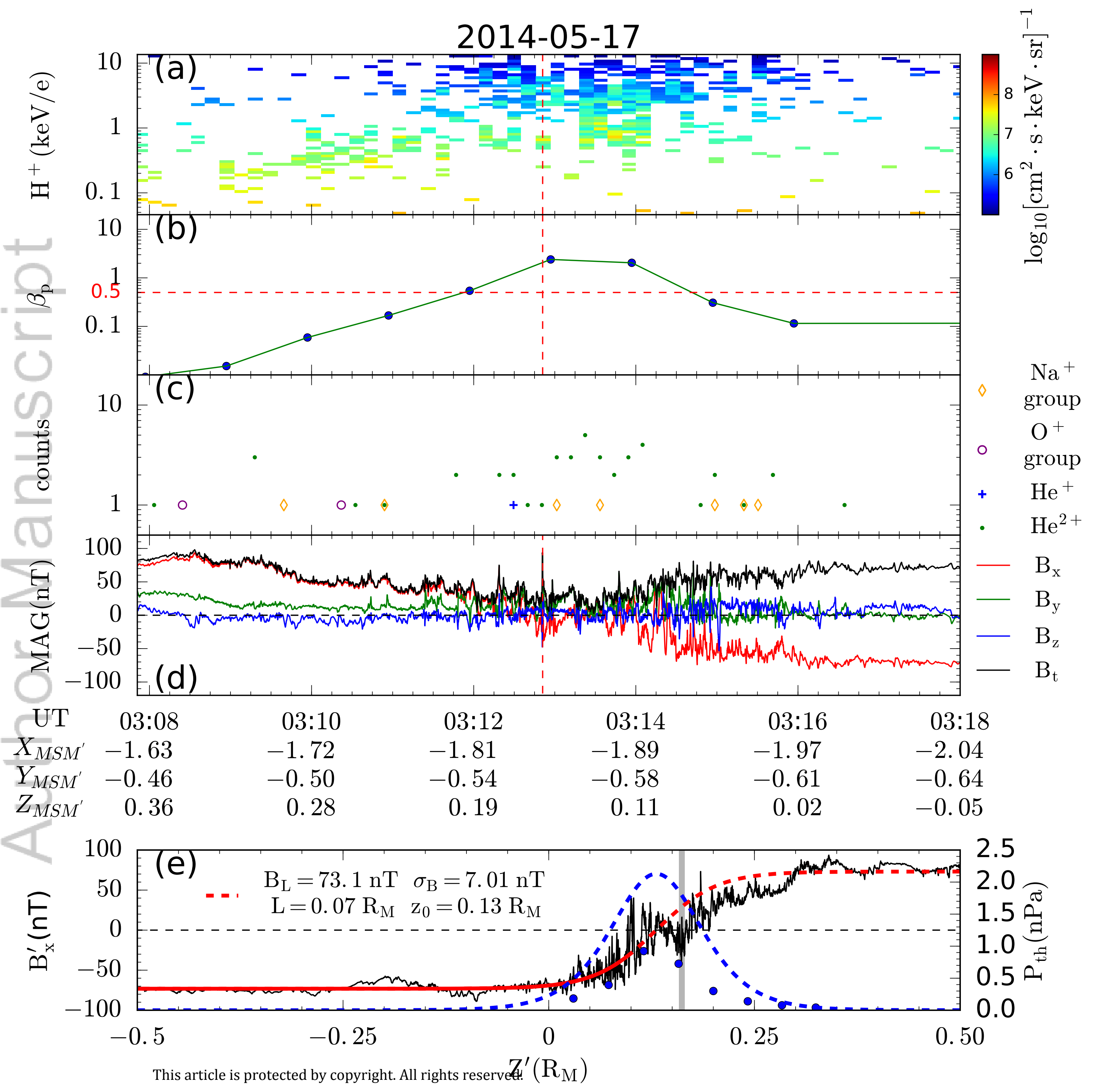



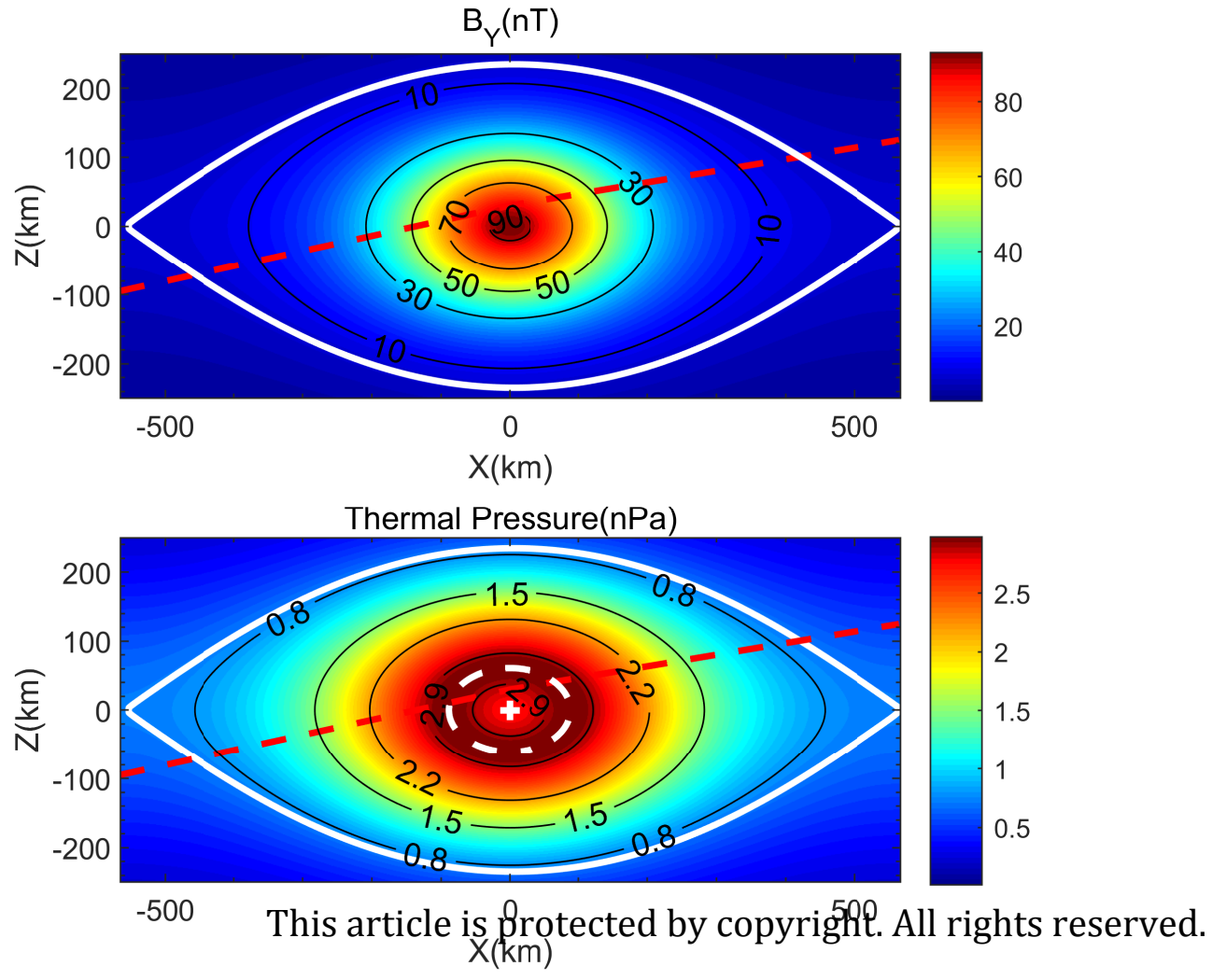

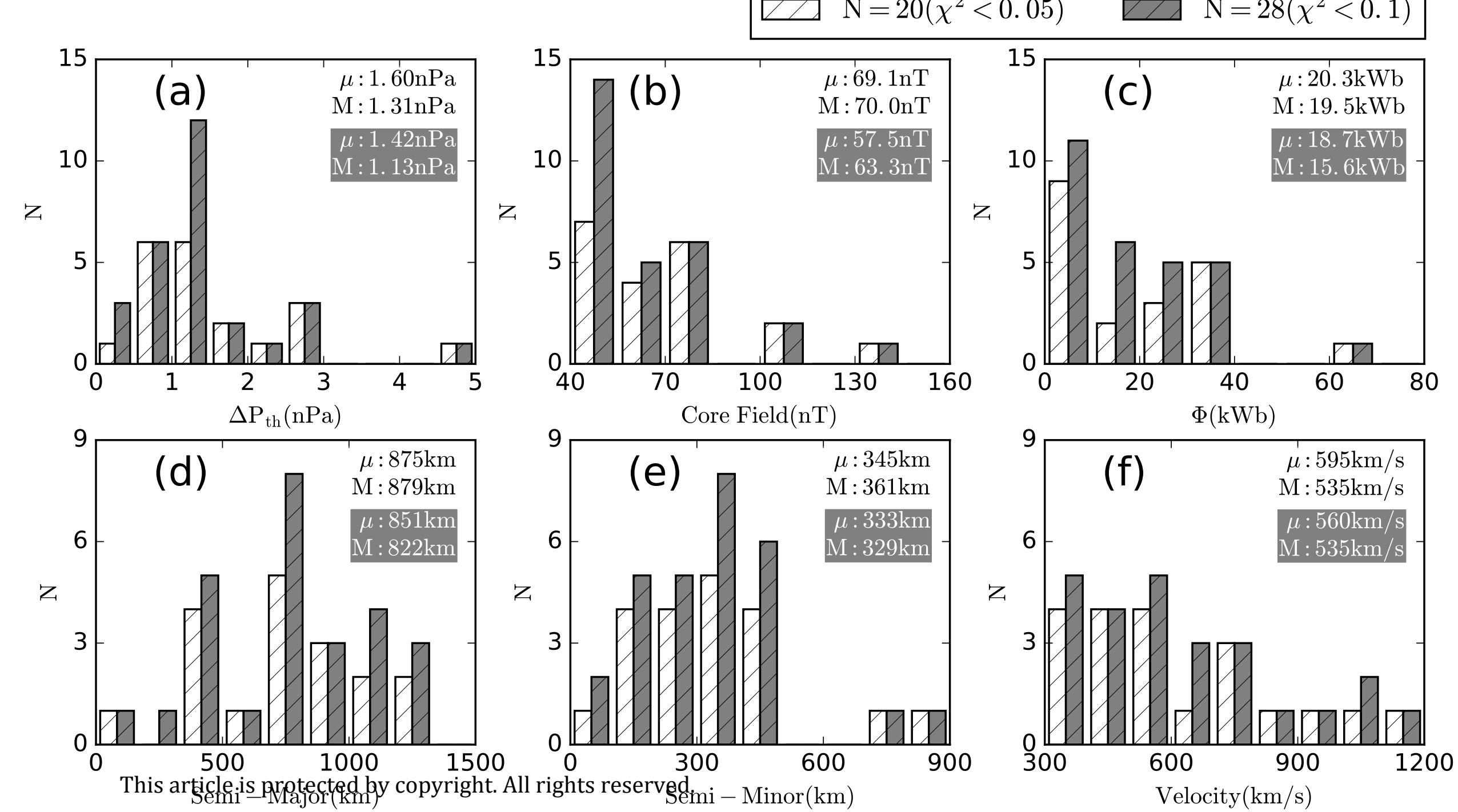


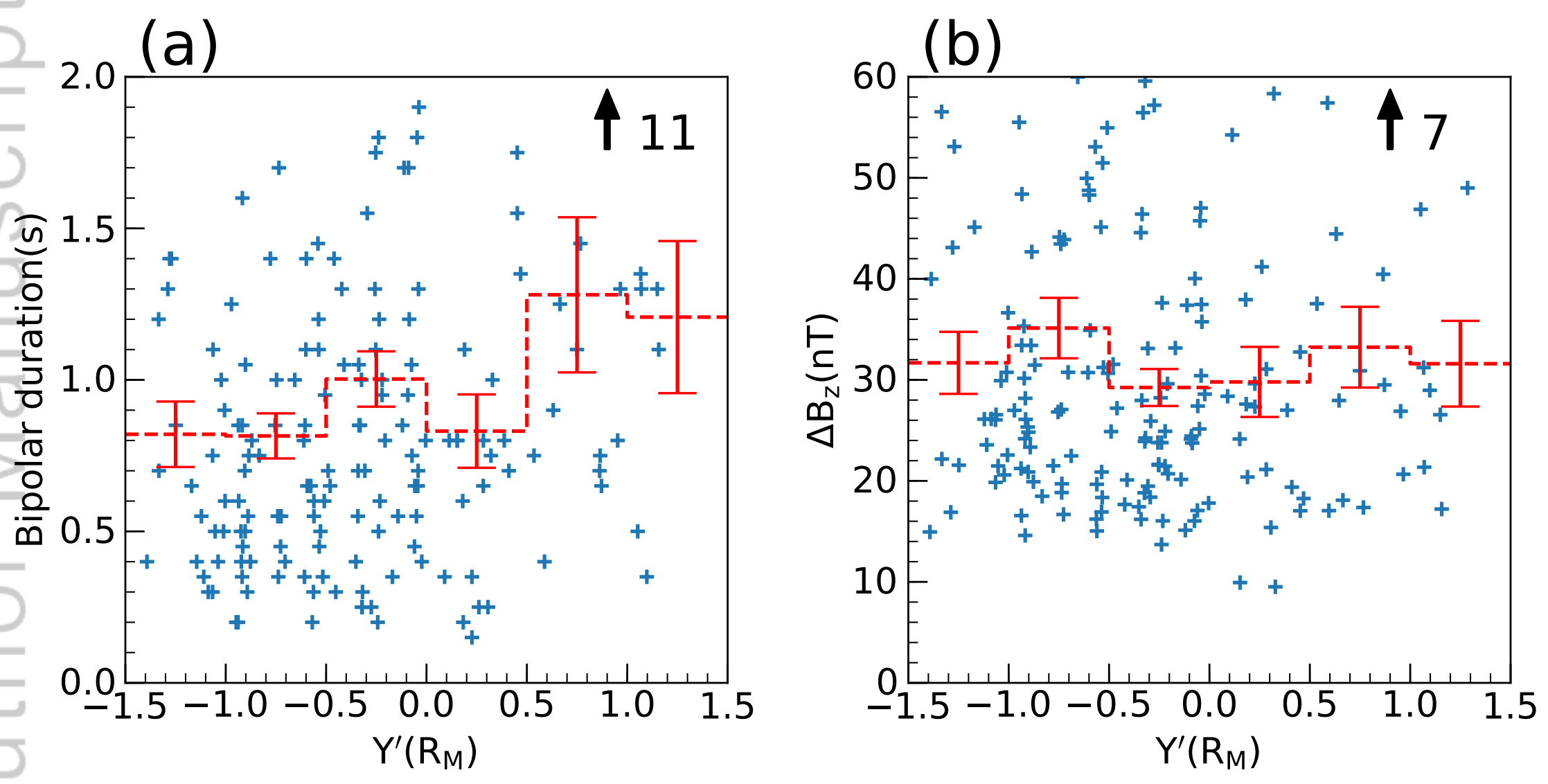

This article is protected by copyright. All rights reserved. 
Flux Rope-2014-05-17
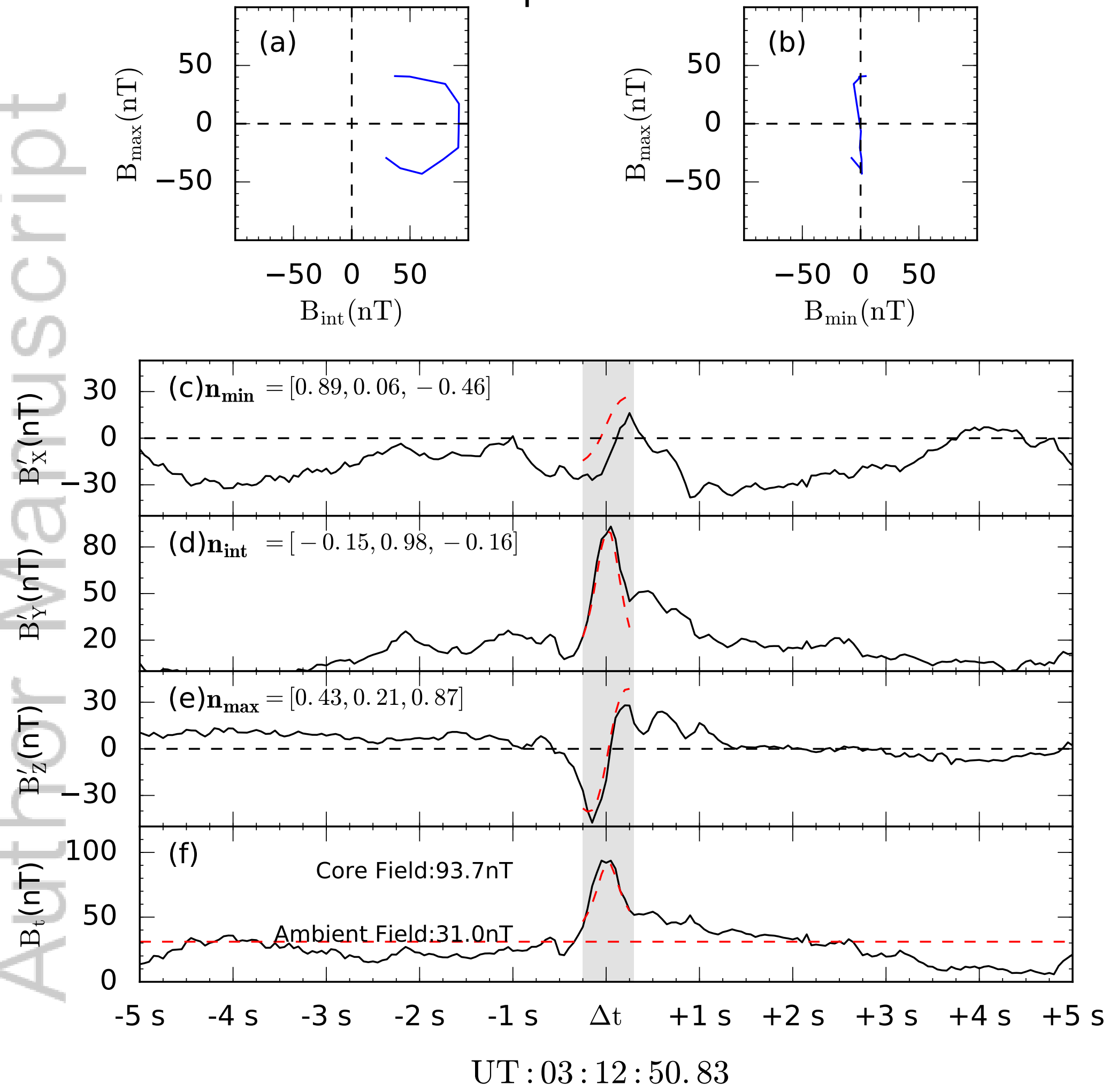

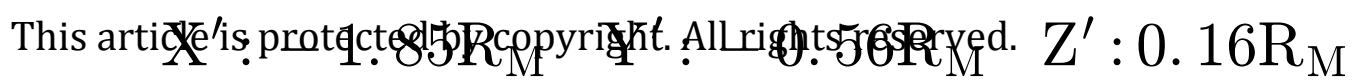




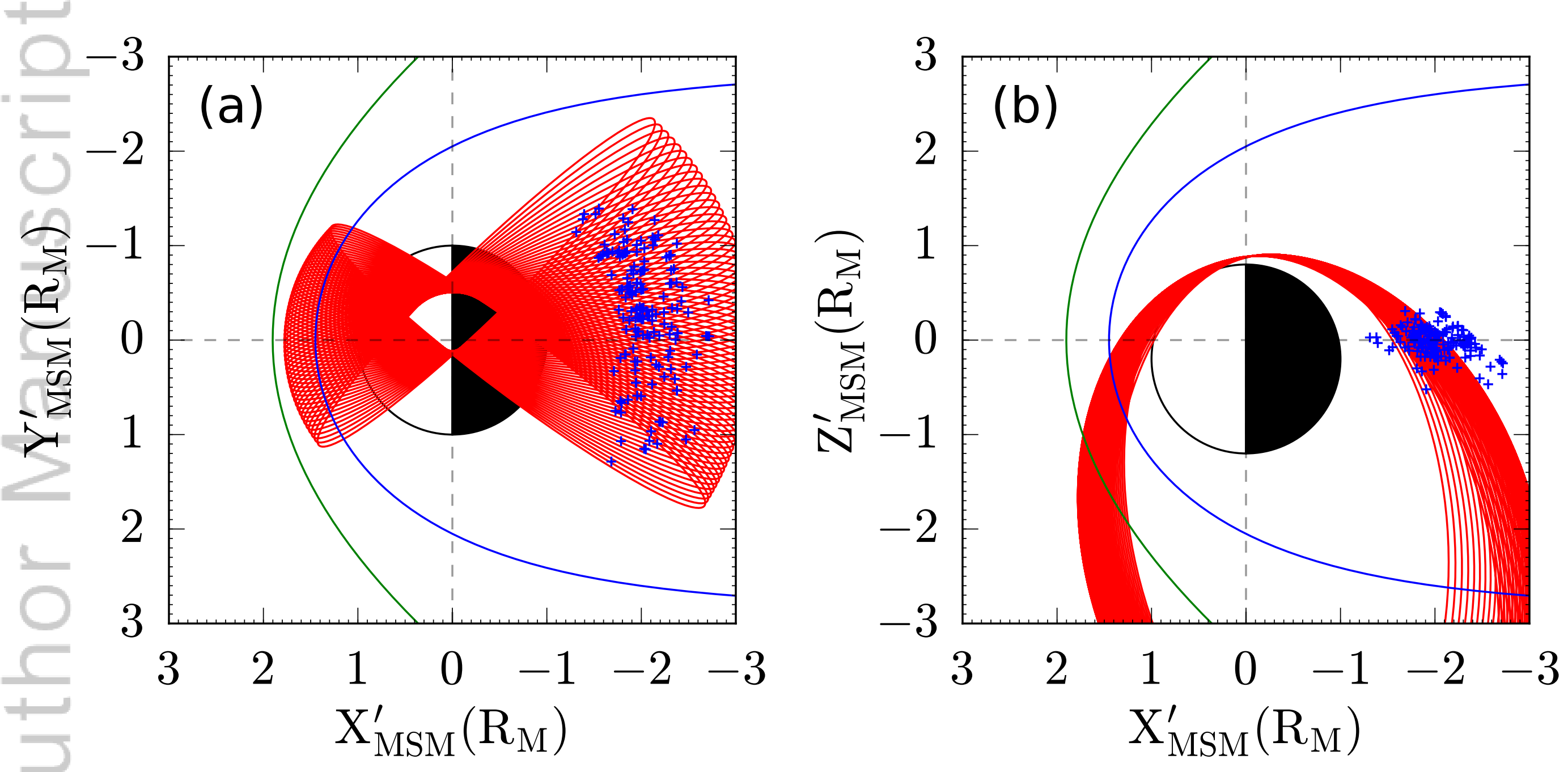

This article is protected by copyright. All rights reserved. 

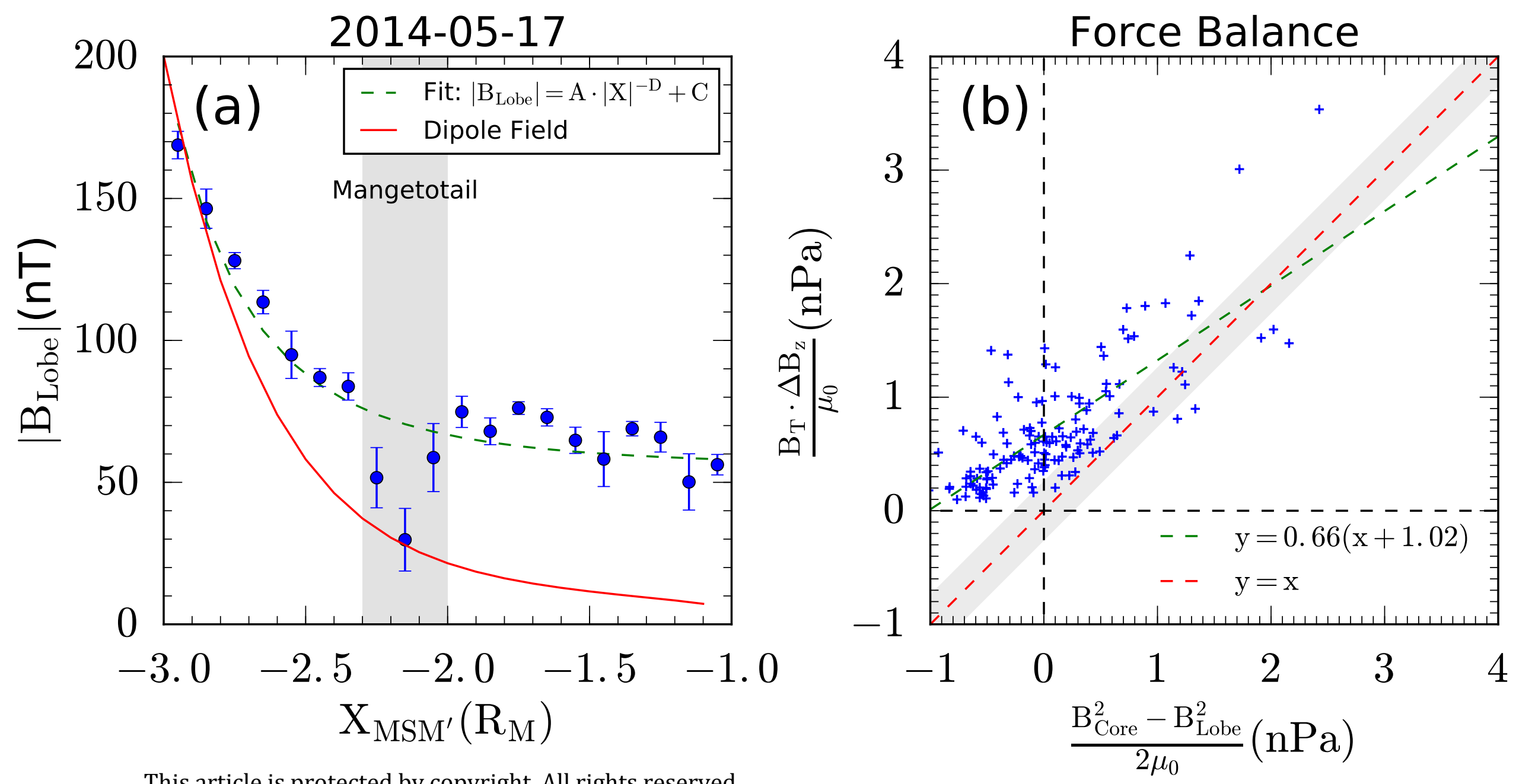

This article is protected by copyright. All rights reserved. 


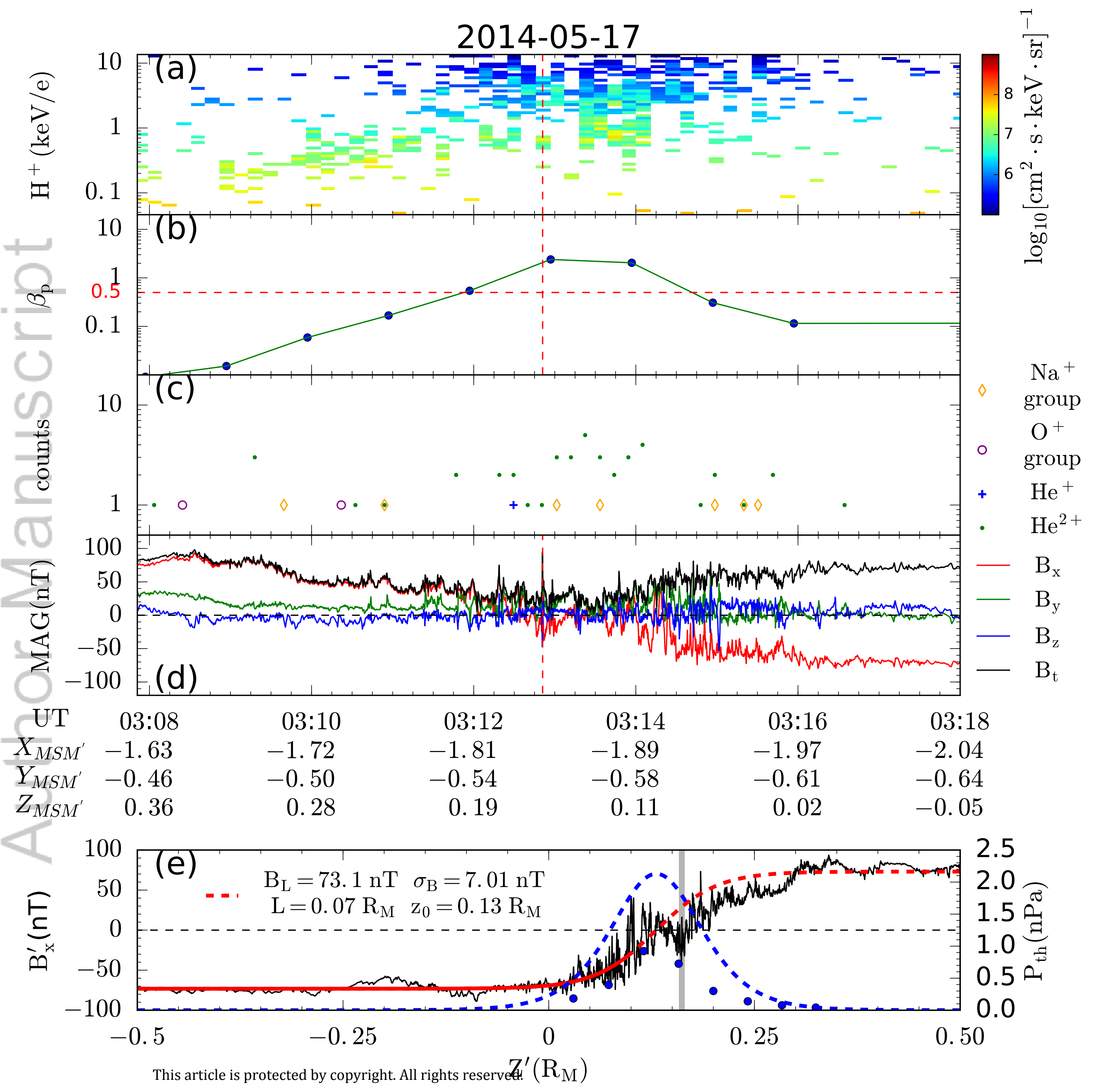



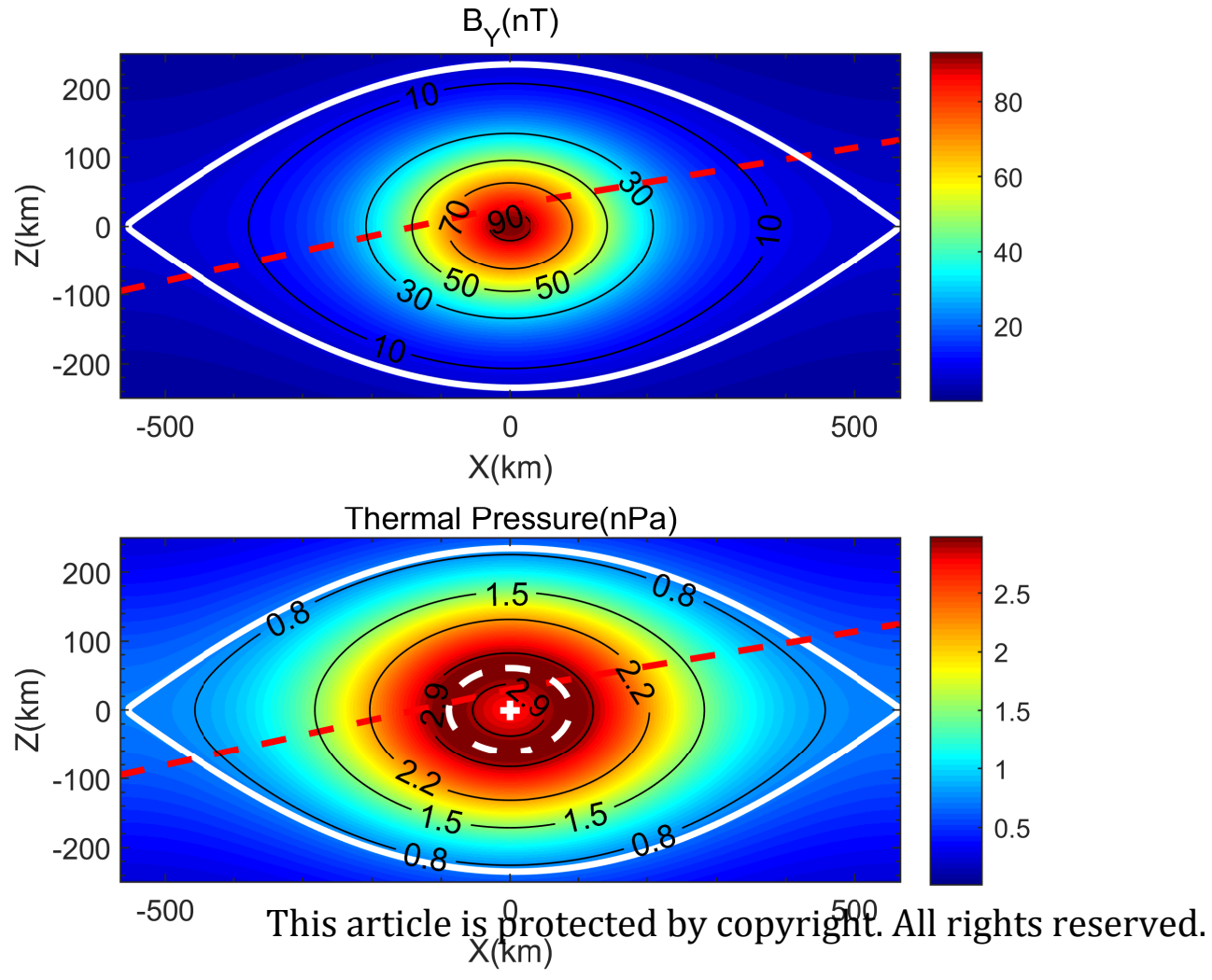

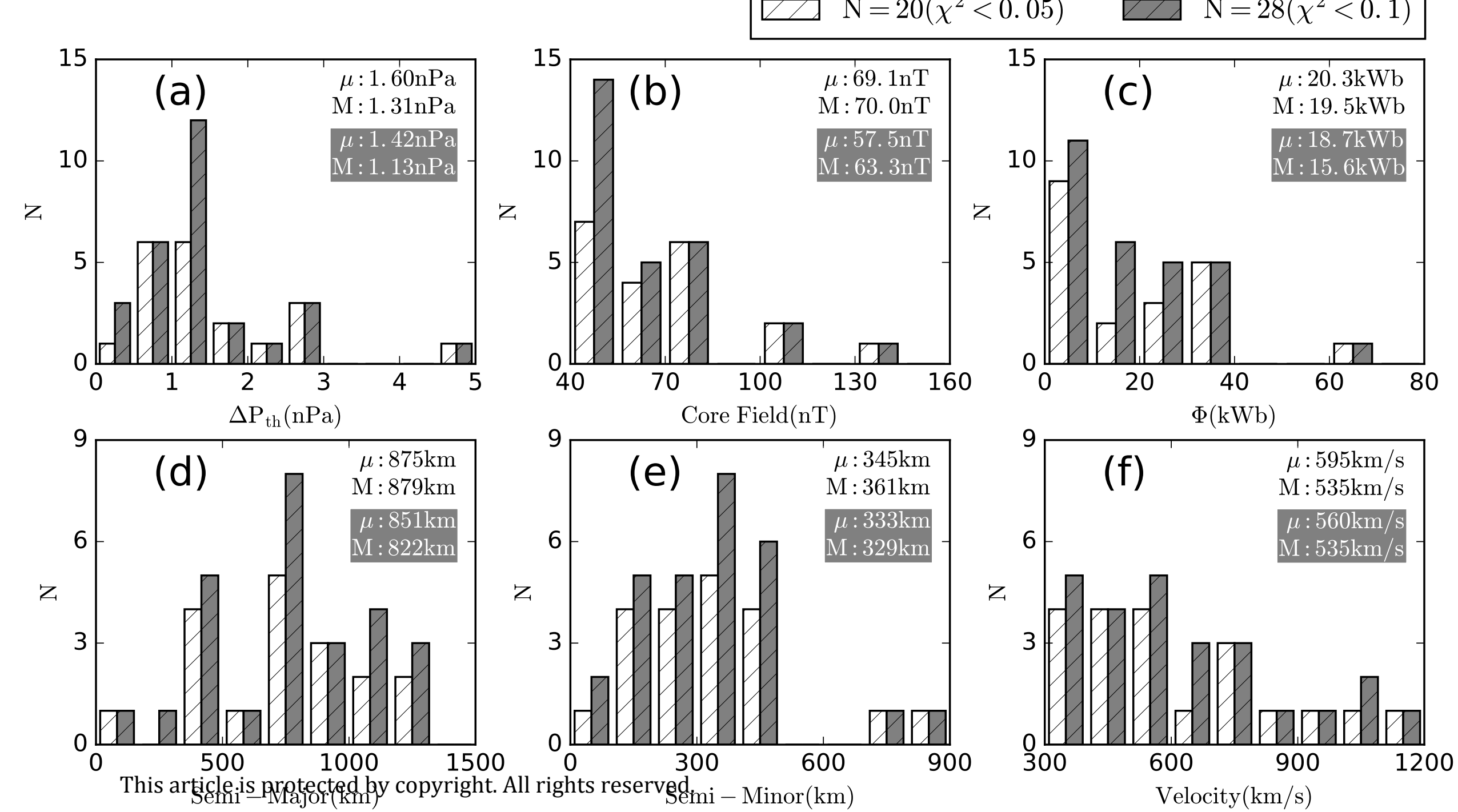


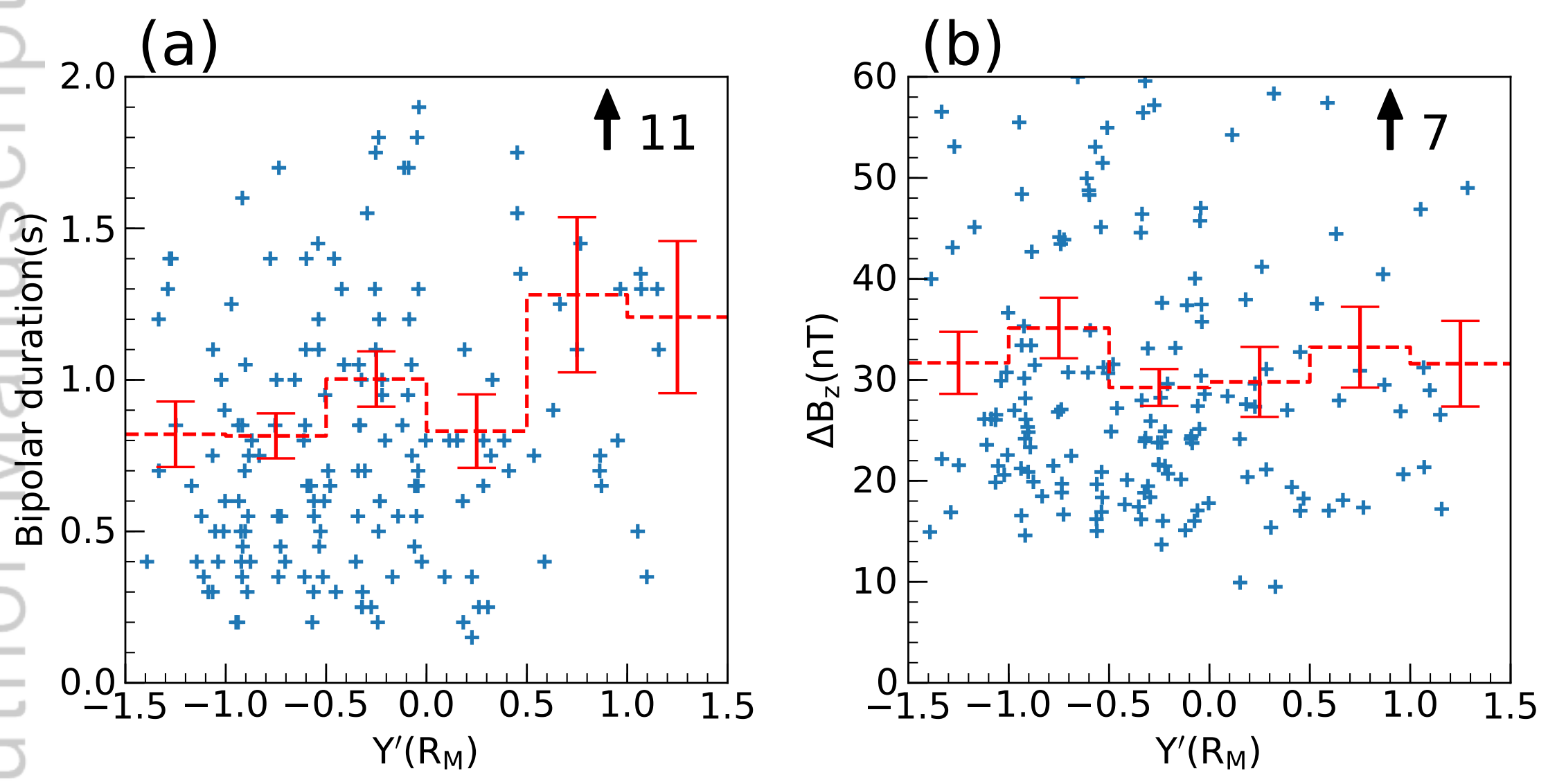

This article is protected by copyright. All rights reserved. 